\title{
Aminotroponiminates: Alkali Metal Compounds Reveal Unprecedented Coordination Modes
}

\author{
Crispin Lichtenberg* \\ Institut für Anorganische Chemie, Julius-Maximilians-Universität Würzburg, Am Hubland, \\ 97074 Würzburg (Germany)
}

\section{Table of contents}

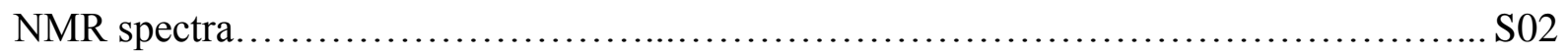

${ }^{7} \mathrm{Li}$ NMR chemical shifts of $\mathbf{2}$ in different solvents................................ 12

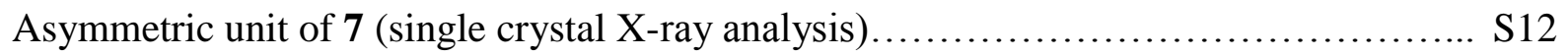

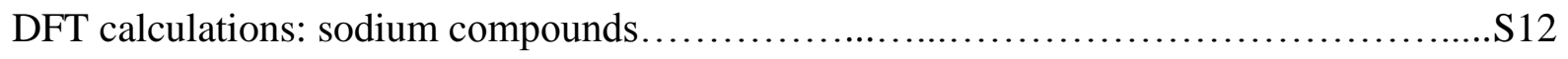

DFT calculations: rhodium compounds........................................... 13

DFT calculations: tungsten compounds......................................... 14

DFT calculations: sterically encumbered Rh ATI compounds..........................S16

Cartesian coordinates and energies of calculated compounds............................S18

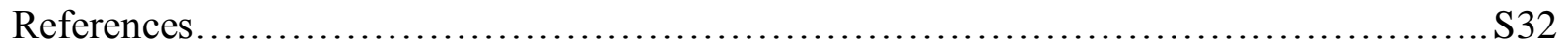



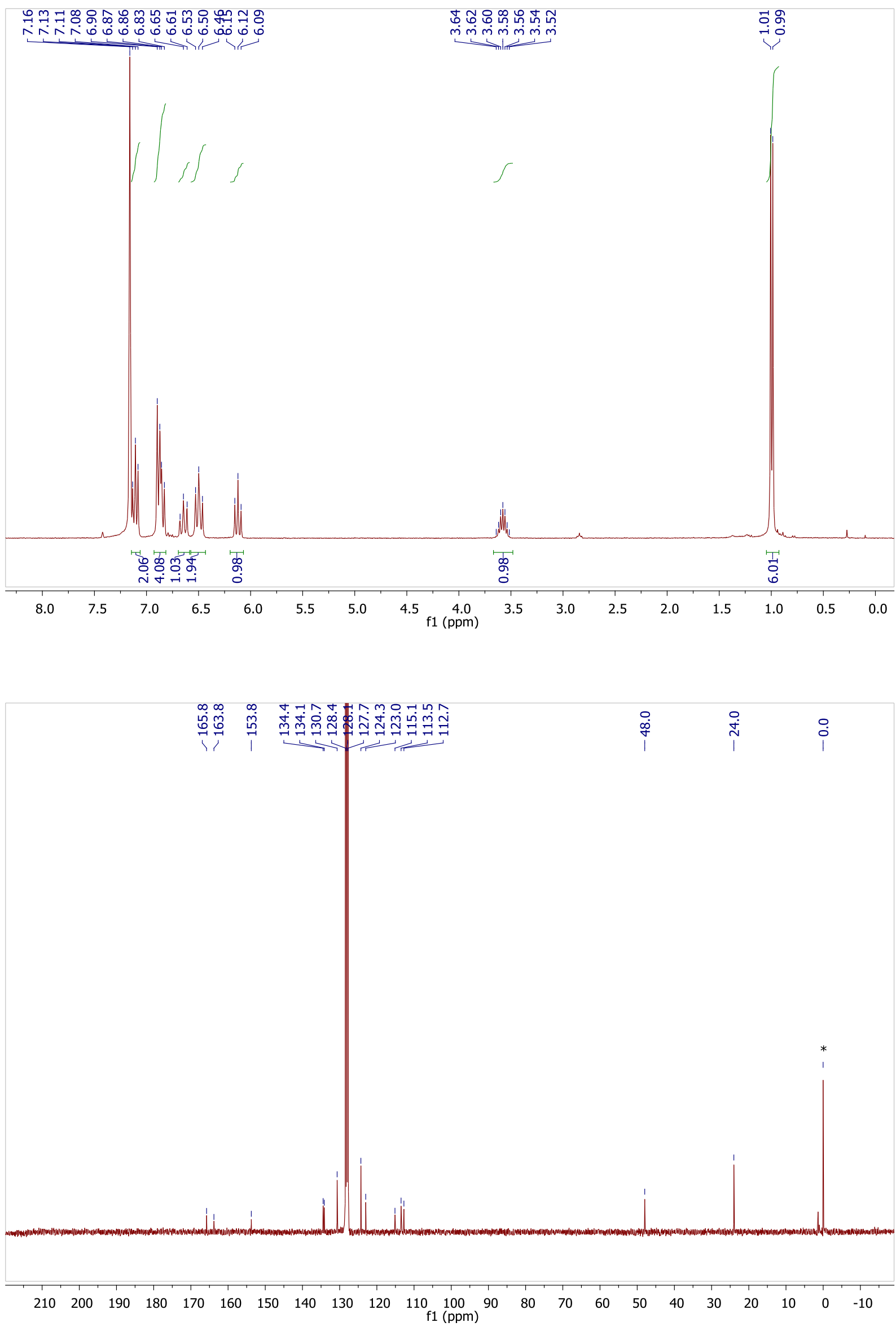

Figure S1a. ${ }^{1} \mathrm{H}$ and ${ }^{13} \mathrm{C}$ NMR spectra of 2 in $\mathrm{C}_{6} \mathrm{D}_{6}$. The resonance in the ${ }^{13} \mathrm{C}$ NMR spectrum marked with an asterisk is due to $\mathrm{SiMe}_{4}$. 


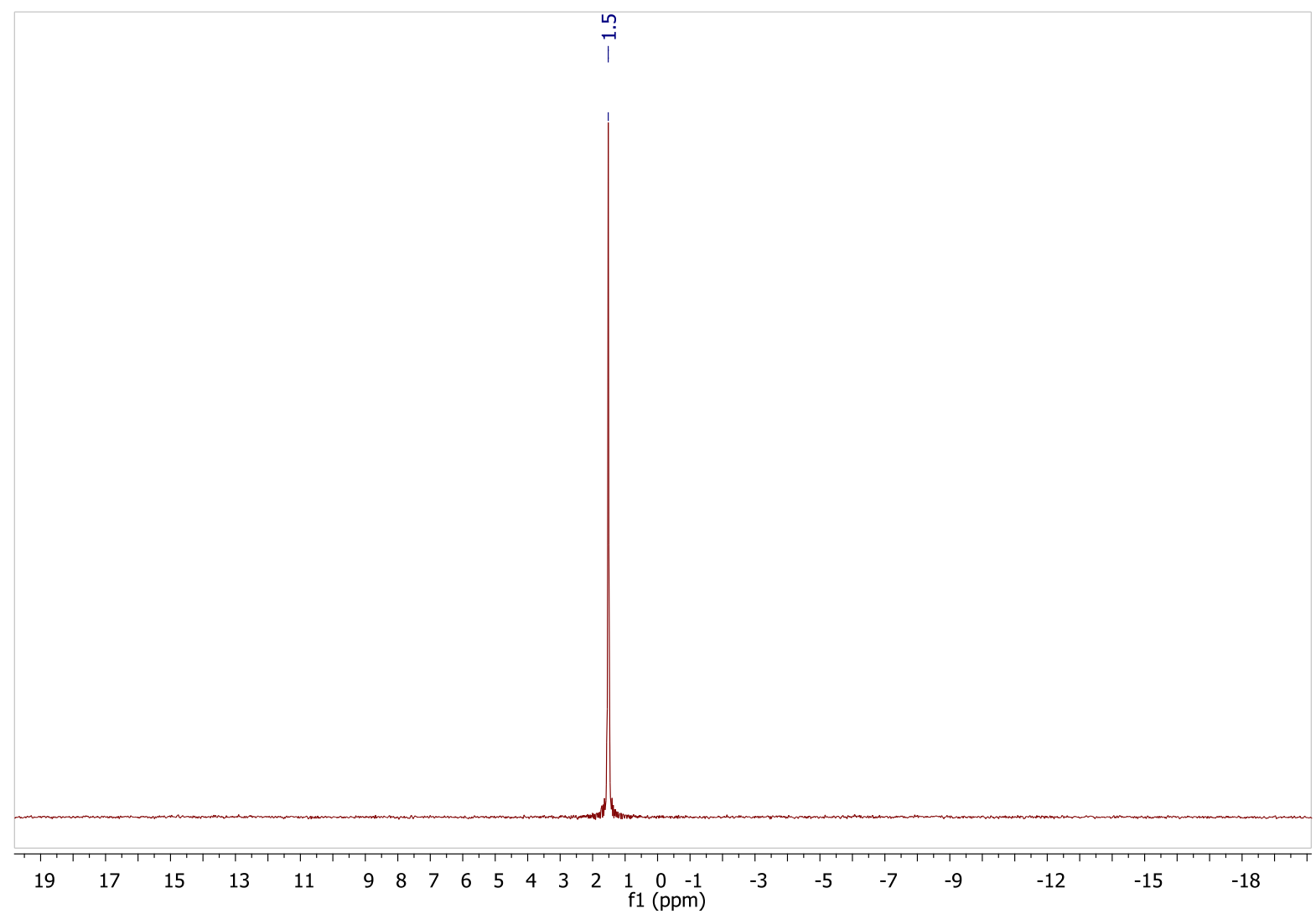

Figure S1b. ${ }^{7} \mathrm{Li}$ NMR spectrum of 2 in $\mathrm{C}_{6} \mathrm{D}_{6}$. 

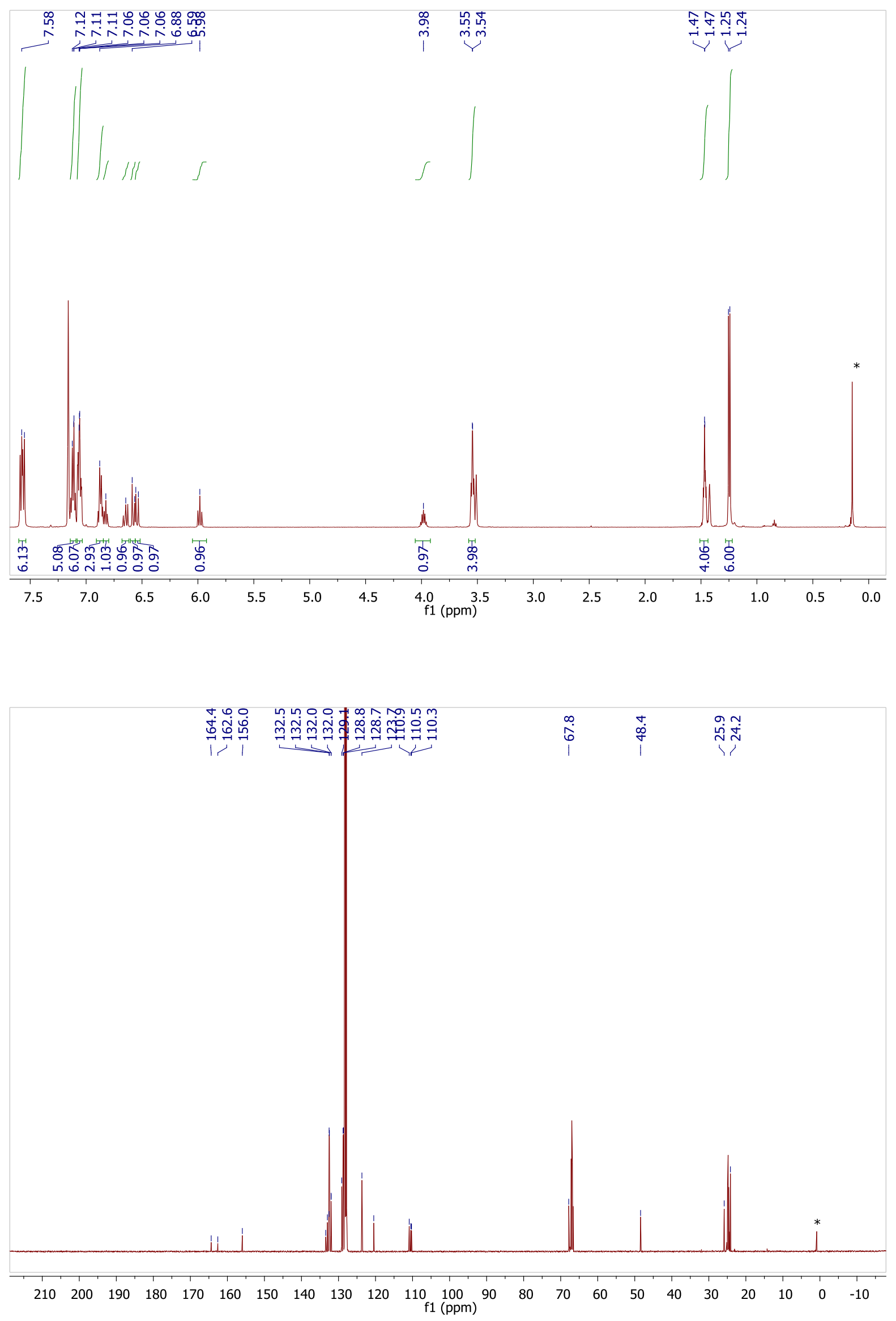

Figure S2a. ${ }^{1} \mathrm{H}$ and ${ }^{13} \mathrm{C}$ NMR spectra of 2-thf-OPPh 3 in $\mathrm{C}_{6} \mathrm{D}_{6} / \mathrm{THF}-d_{8}(5: 1)$. The resonances marked with an asterisk are due to silicon grease. 

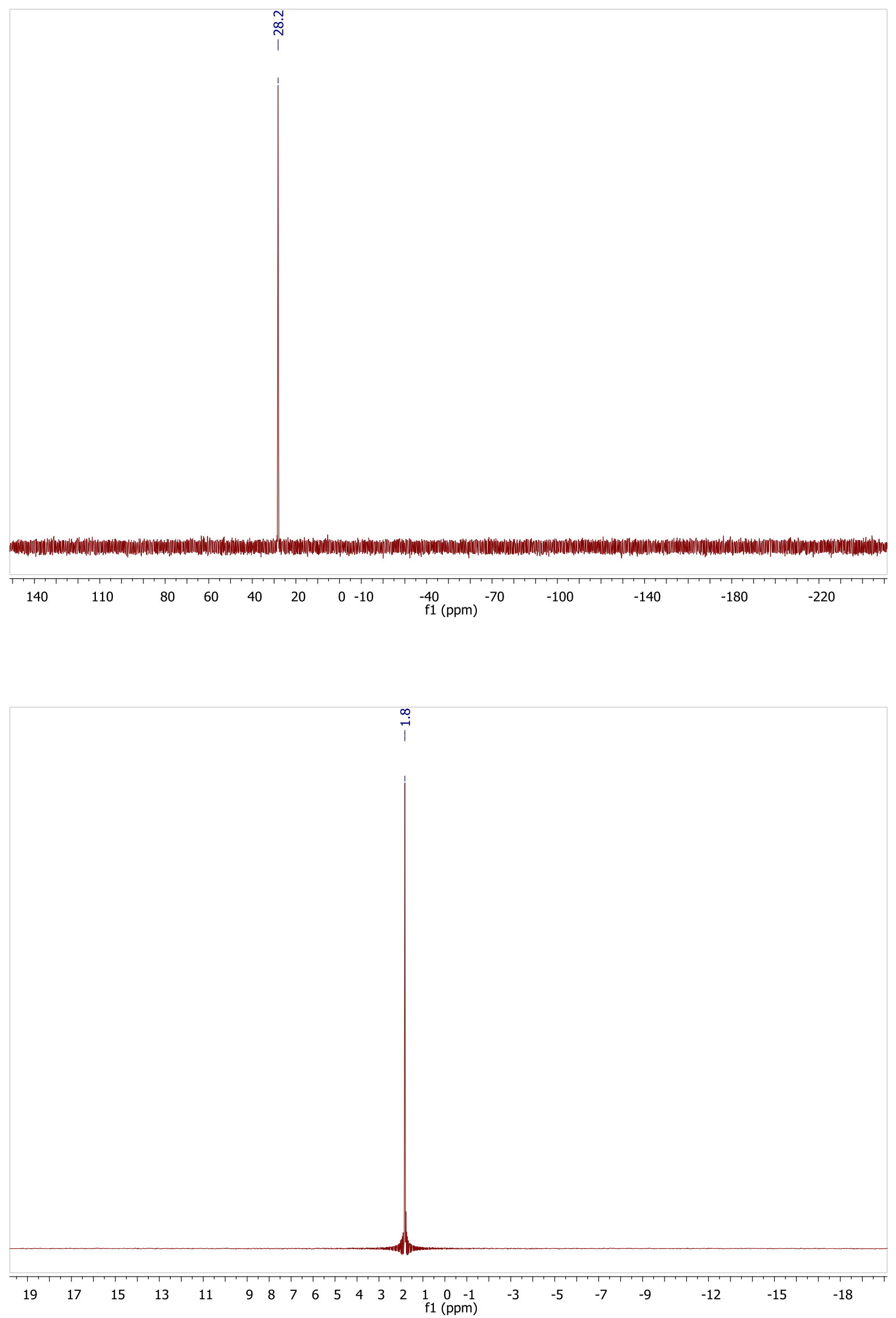

Figure S2b. ${ }^{31} \mathrm{P}$ and ${ }^{7} \mathrm{Li}$ NMR spectra of 2-thf-OPPh 3 in $\mathrm{C}_{6} \mathrm{D}_{6} / \mathrm{THF}-d_{8}(5: 1)$. 

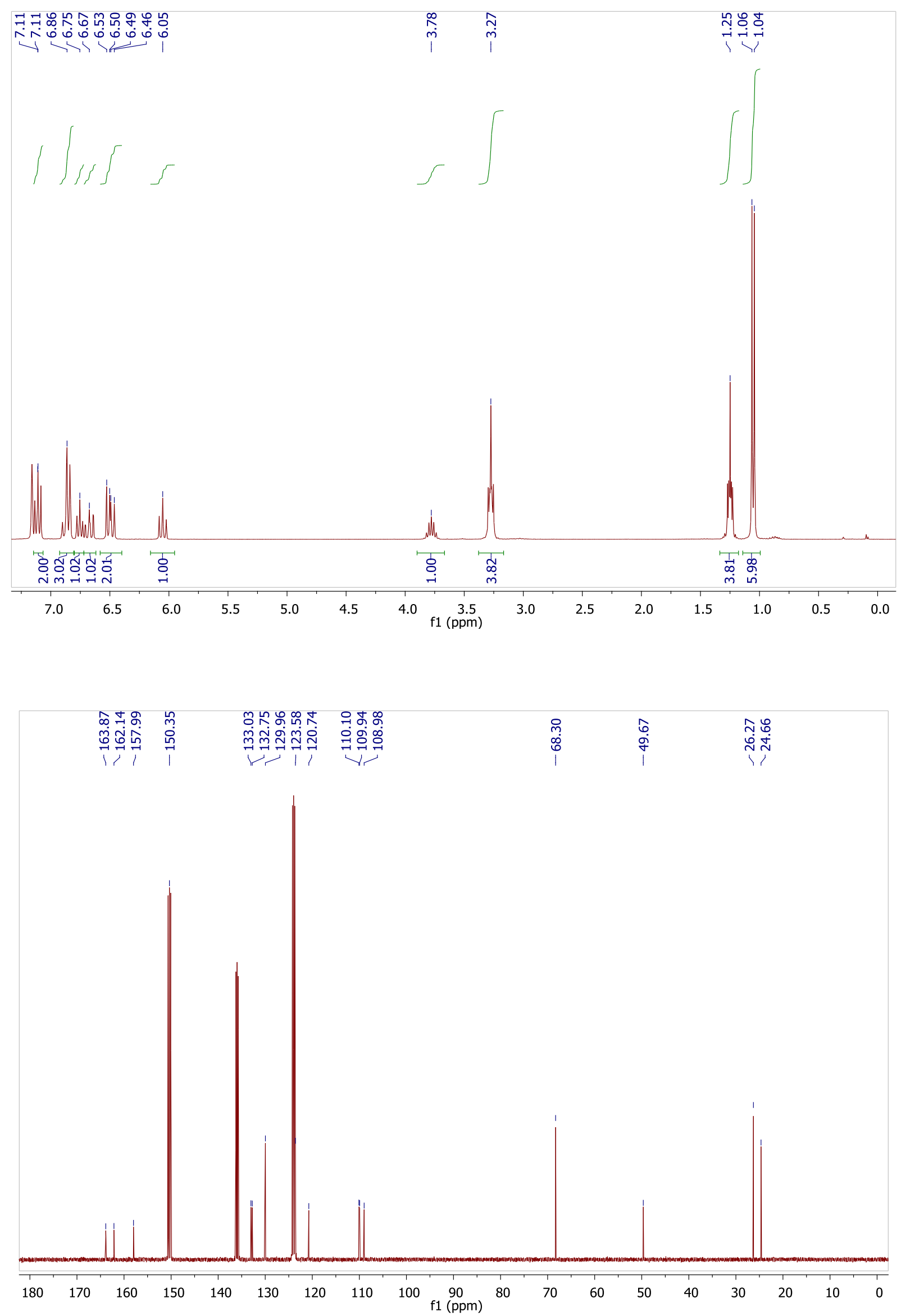

Figure S3a. ${ }^{1} \mathrm{H}$ and ${ }^{13} \mathrm{C}$ NMR spectra of 3-thf in $\mathrm{C}_{6} \mathrm{D}_{6}$ and $\mathrm{C}_{5} \mathrm{D}_{5} \mathrm{~N}$, respectively. 

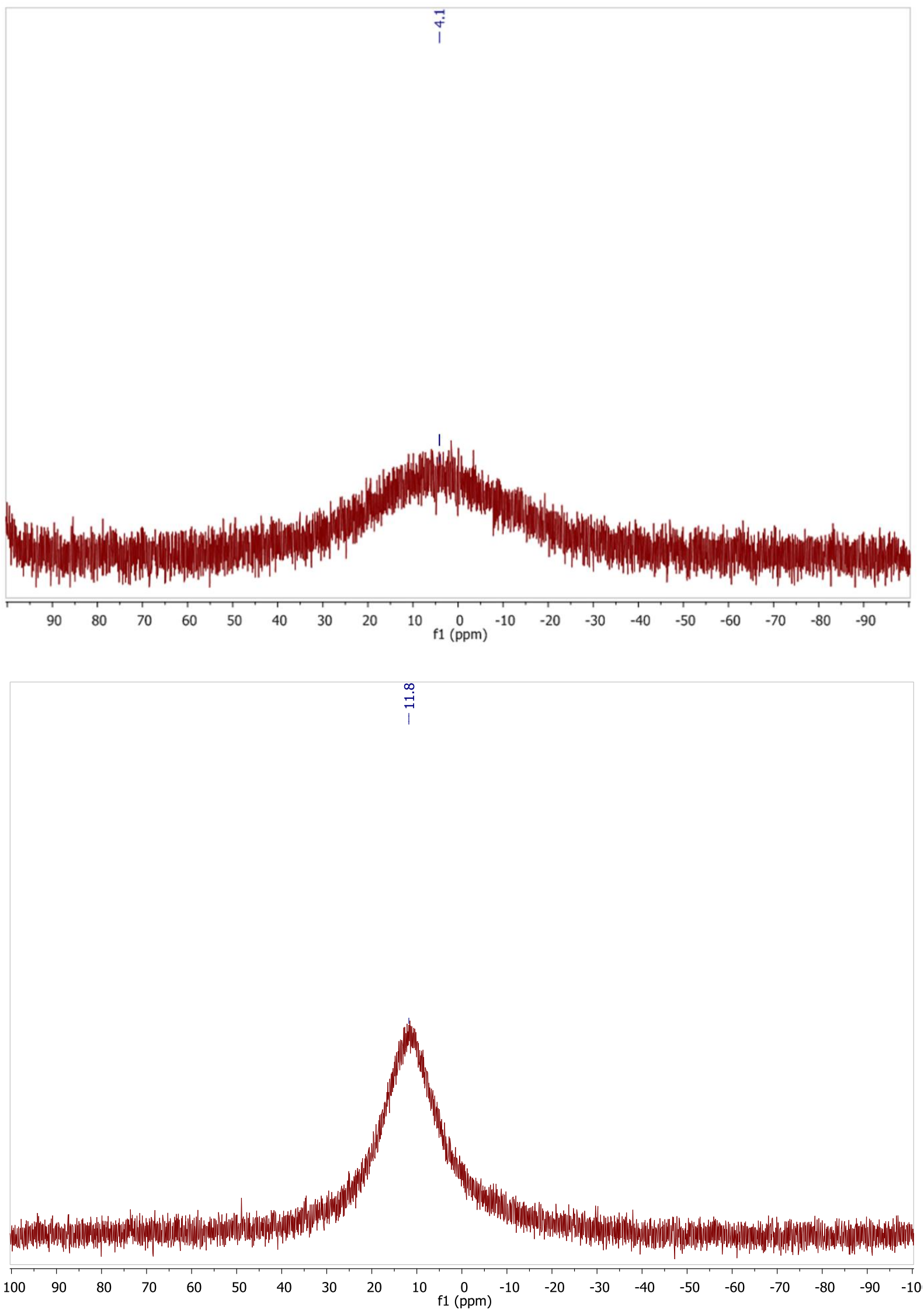

Figure S3b. ${ }^{23} \mathrm{Na}$ NMR spectra of 3-thf in $\mathrm{C}_{6} \mathrm{D}_{6}$ (top) and $\mathrm{C}_{5} \mathrm{D}_{5} \mathrm{~N}$ (bottom). Background signals from NMR tubes were insignificant as determined by measurements of NMR tubes without samples. 

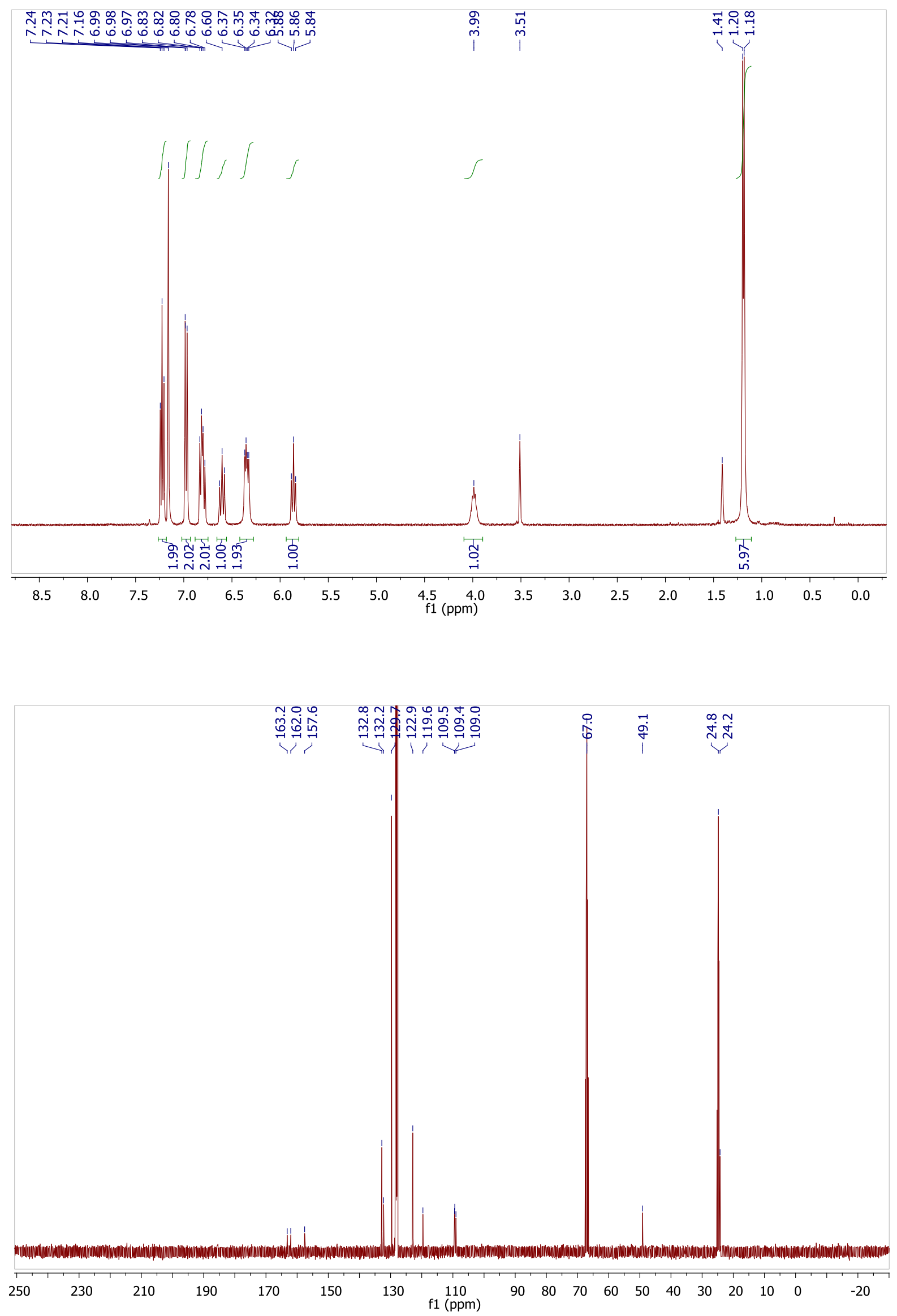

Figure S4. ${ }^{1} \mathrm{H}$ and ${ }^{13} \mathrm{C}$ NMR spectra of 4 (solvent free form) in $\mathrm{C}_{6} \mathrm{D}_{6} / \mathrm{THF}-d_{8}$ (5:1). NMR spectra of 4-thf are identical since THF- $d_{8}$ is present as a solvent. 

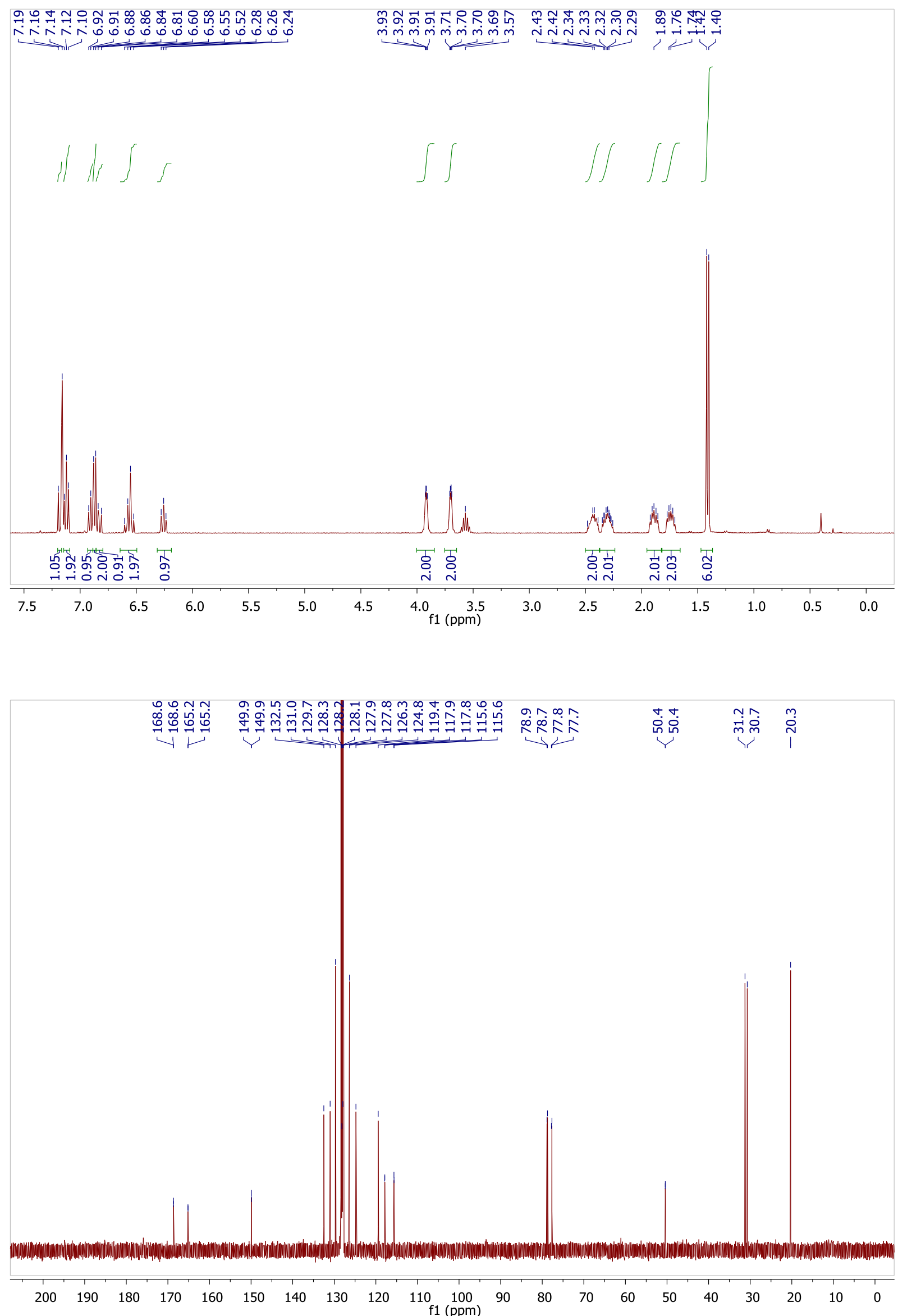

Figure S5. ${ }^{1} \mathrm{H}$ and ${ }^{13} \mathrm{C}$ NMR spectra of 5 in $\mathrm{C}_{6} \mathrm{D}_{6}$. 

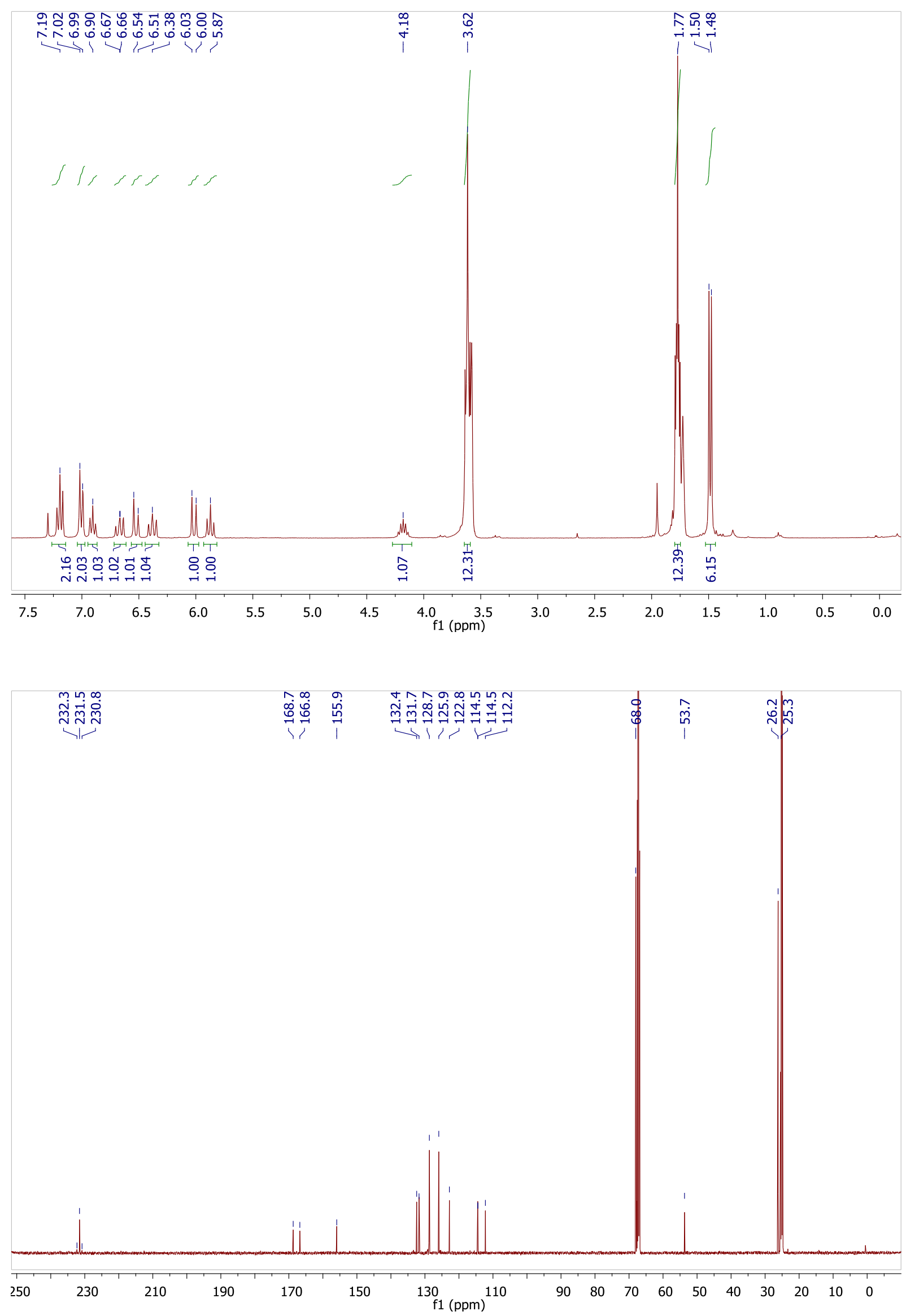

Figure S6. ${ }^{1} \mathrm{H}$ and ${ }^{13} \mathrm{C}$ NMR spectra of 6 in THF- $d_{8}$. 


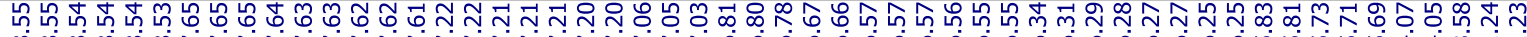

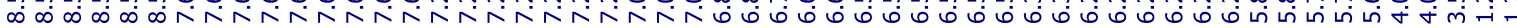
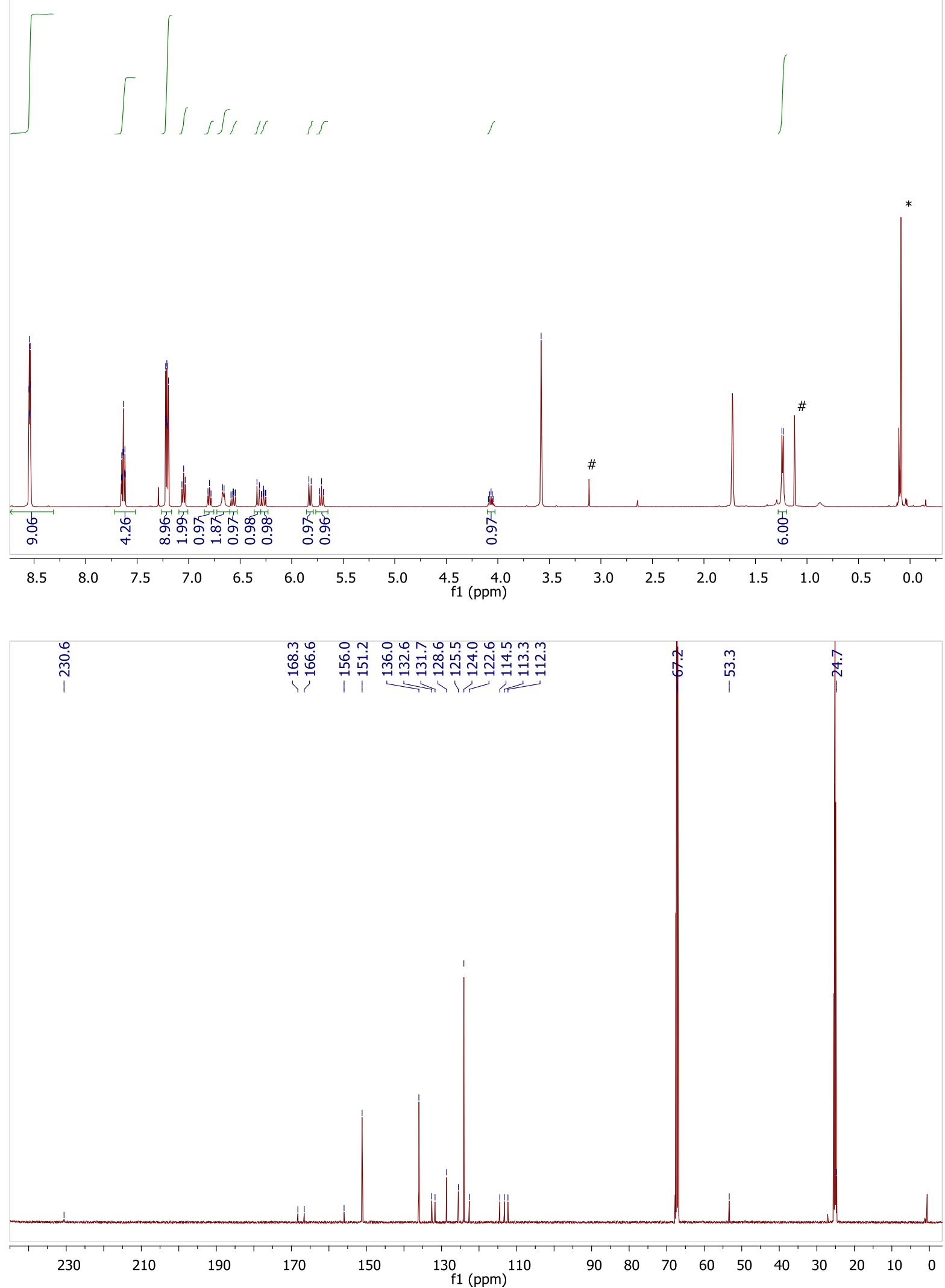

Figure S7. ${ }^{1} \mathrm{H}$ and ${ }^{13} \mathrm{C}$ NMR spectra of 7 in THF- $d_{8}$ at $40{ }^{\circ} \mathrm{C}$. Resonances marked with a hashtag or an asterisk are due to traces of MTBE and silicon grease, respectively. 
Table S1. ${ }^{7} \mathrm{Li}$ NMR chemical shifts for $\mathbf{2}$ in the absence or presence of neutral donor ligands.

\begin{tabular}{c|ccc}
\hline $\mathbf{L 1} / \mathbf{L 2}$ & - & $\mathrm{THF} / \mathrm{THF}$ & $\mathrm{THF} \mathrm{OPPh}_{3}$ \\
Solvent & $\mathrm{C}_{6} \mathrm{D}_{6}$ & $\mathrm{THF}-d_{8}$ & $\mathrm{C}_{6} \mathrm{D}_{6} / \mathrm{THF}-d_{8}(5: 1)$ \\
${ }^{{ }^{7} \mathbf{L i} \boldsymbol{\delta} \text { [ppm] }}$ & 1.52 & 1.50 & 1.83 \\
\hline
\end{tabular}

Single crystal X-ray analysis of 7. The asymmetric unit of $\mathbf{7}$ consists of two formula units of $\left[\mathrm{NaW}\left(\mathrm{ATI}^{\mathrm{Ph} / \mathrm{iPr}}\right)(\mathrm{CO})_{3}(\mathrm{py})_{4}\right]$, three lattice bound molecules of pyridine and one lattice bound molecule of MTBE (Figure S8). Due to severe crystallographic disorder of lattice bound solvent molecules, they were refined isotropically and restraints and constraints were applied during refinement. Restraints and constraints were also applied during refinement of some of the sodium bound pyridine molecules due to unresolved crystallographic disorder.

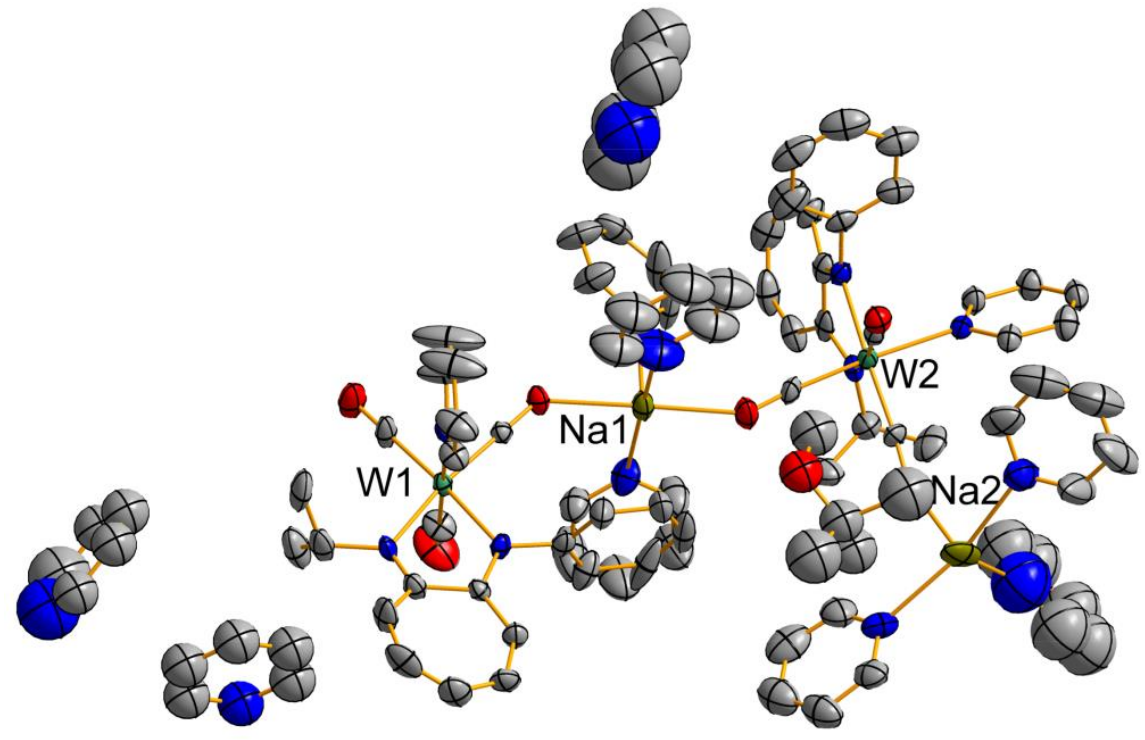

Figure S8. Asymmetric unit of 7. Displacement ellipsoids are shown at the 50\% probability level; hydrogen atoms are omitted for clarity.

\section{DFT calculations: sodium compounds}
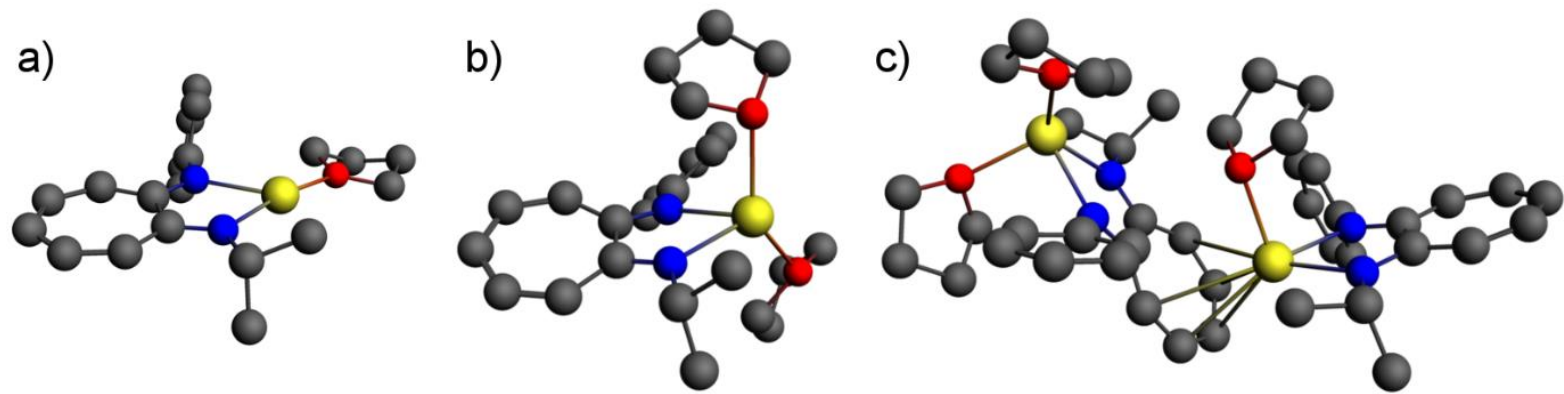

Figure S9 Calculated structures of a) $\left[\mathrm{Na}\left(\mathrm{ATI}^{\mathrm{Ph} / \mathrm{Pr}}\right)(\mathrm{thf})\right]$, b) $\left[\mathrm{Na}\left(\mathrm{ATI}^{\mathrm{Ph} / \mathrm{Pr}}\right)(\mathrm{thf})_{2}\right]$, and c) $\left[\mathrm{Na}_{2}\left(\mathrm{ATI}^{\mathrm{Ph} / \mathrm{Pr}}\right)_{2}(\mathrm{thf})_{3}\right](\mathbf{8})$; color code for atoms: grey $=\mathrm{C}$; blue $=\mathrm{N}$; red $=\mathrm{O}$; yellow $=\mathrm{Na}$. 
Comparison of 3-thf (exp.) and 8 (calc.). The dimer 8 was calculated in the gas phase as a model for the polymeric structure of 3-thf in the solid state. One of the sodium atoms in $\mathbf{8}$ shows a coordination geometry similar to that of the sodium atoms in 3-thf. The bond lengths involving these sodium atoms are summarized in Table S2. The calculated values are in fair agreement with the experimentally determined distances. A slight underestimation of calculated $\mathrm{Na}-\mathrm{N}$ and a slight overestimation of calculated $\mathrm{Na}-\mathrm{O}$ and $\mathrm{Na}-\mathrm{C}$ bond distances is observed. Moreover, the sodium atom in the calculated structure is marginally shifted towards one side of the ATI ligand backbone, which leads to significantly larger values for calculated Na1-C6'/C7' distances.

Table S2. Comparison of bond lengths involving Na1 of 3-thf (experimental, solid state, $\mathrm{XRD}$ ) and 8 (calculated, gas phase); Atom labels according to Fig. 2 in main text.

\begin{tabular}{l|cc}
\hline & $\begin{array}{c}\text { Compound 3-thf (experimental, XRD) } \\
{[\AA]}\end{array}$ & $\begin{array}{c}\text { Compound 8 (calculated) } \\
{[\AA]}\end{array}$ \\
\hline Na1-N1 & $2.3643(15)$ & 2.3387 \\
Na1-N2 & $2.3563(15)$ & 2.3163 \\
Na1-O1 & $2.3047(14)$ & 2.4356 \\
Na1-C3, & $3.1071(18)$ & 3.1664 \\
Na1-C4, & $2.9313(19)$ & 3.0460 \\
Na1-C5, & $2.8034(18)$ & 3.0217 \\
Na1-C6, & $2.7957(18)$ & 3.1321 \\
Na1-C7, & $3.0604(18)$ & 3.4146 \\
\hline
\end{tabular}

DFT Calculations: rhodium compounds
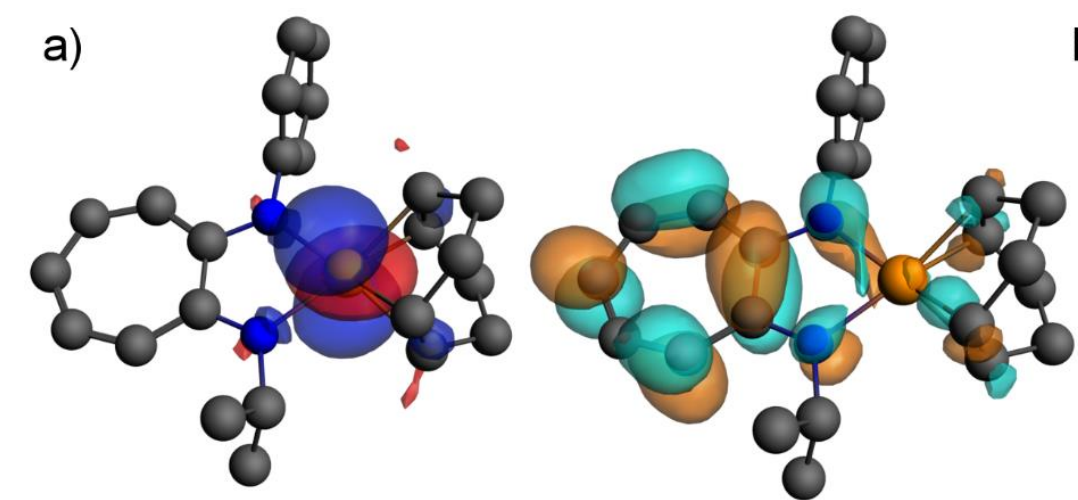

b)

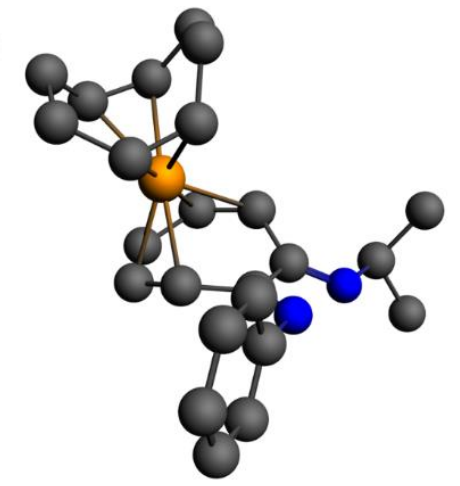

Figure S10. a) Calculated structure of $\left[\mathrm{Rh}\left(\mathrm{ATI}^{\mathrm{Ph} / \mathrm{Pr}}-\kappa^{2} N\right)(\operatorname{cod})\right](5)$ with HOMO (left) and LUMO (right) at isovalues of 0.03 ; b) calculated structure of $\left[\mathrm{Rh}\left(\mathrm{ATI}^{\mathrm{Ph} / i \mathrm{Pr}}-\kappa^{5} C\right)(\mathrm{cod})\right](\mathbf{5}-\mathbf{p i})$; color code for atoms: grey $=\mathrm{C}$; blue $=\mathrm{N}$; orange $=\mathrm{Rh}$.

Comparison of 5 (exp.) and 5 (calc.). The experimentally determined structure of 5 in the solid state is monomeric. Thus a model compound for DFT calculations with identical connectivities for all atoms could be used. Consequently, the experimentally (solid state XRD) and theoretically (DFT, gas phase) determined bonding parameters of $\mathbf{5}$ are in excellent agreement (Table S3). 
Table S3. Comparison of bond lengths involving Rh1 of 5 (experimental, solid state, XRD) and 5 (calculated, gas phase); Atom labels according to Fig. 4 in main text.

\begin{tabular}{|c|c|c|}
\hline & $\begin{array}{c}\text { Compound } 5 \text { (experimental, XRD) } \\
{[\stackrel{\AA}{A}]}\end{array}$ & $\begin{array}{c}\text { Compound } 5 \text { (calculated) } \\
{[\AA \AA]}\end{array}$ \\
\hline Rh1-N1 & $2.070(5)$ & 2.0701 \\
\hline Rh1-N2 & $2.023(6)$ & 2.0381 \\
\hline Rh1-C17 & $2.134(6)$ & 2.1249 \\
\hline Rh1-C18 & $2.143(6)$ & 2.1513 \\
\hline Rh1-C21 & $2.126(5)$ & 2.1343 \\
\hline Rh1-C22 & $2.144(5)$ & 2.1570 \\
\hline
\end{tabular}

DFT calculations: tungsten compounds
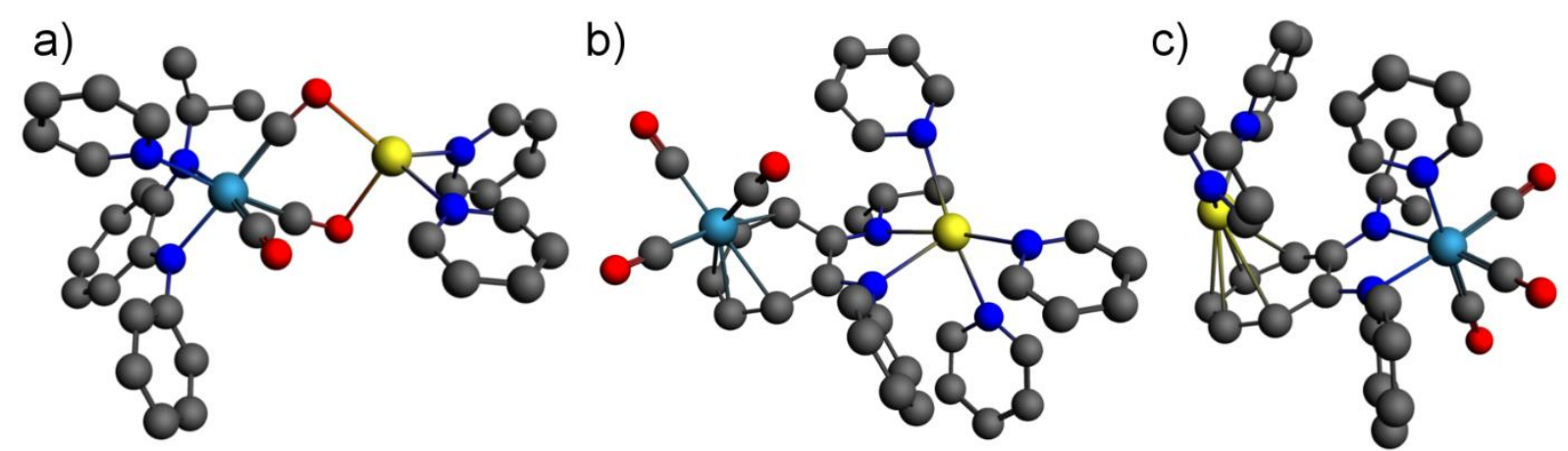

Figure S11. Calculated structures of a) $\left[\mathrm{NaW}\left(\mathrm{ATI}^{\mathrm{Ph} / \mathrm{Pr}}-2 \kappa^{2} N\right)(\mathrm{py}-2 \kappa N)\left(\mu_{2}-\mathrm{CO}-\right.\right.$ $\left.1 \kappa O, 2 \kappa C)_{2}(\mathrm{CO}-2 \kappa C)(\mathrm{py}-1 \kappa N)_{2}\right](\mathbf{9})$, b) $\left[\mathrm{NaW}\left(\mathrm{ATI}^{\mathrm{Ph} /} / \mathrm{Pr}-1 \kappa^{2} N, 2 \kappa^{5} C\right)(\mathrm{CO}-2 \kappa C)_{3}(\mathrm{py}-1 \kappa N)_{3}\right](\mathbf{1 0})$, and c) $\left[\mathrm{NaW}\left(\mathrm{ATI}{ }^{\mathrm{Ph} / \mathrm{Pr}}-1 \kappa^{5} C, 2 \kappa^{2} N\right)(\mathrm{CO}-2 \kappa C)_{3}(\text { py- } 1 \kappa N)_{2}(\right.$ py $\left.-2 \kappa N)\right]$ (11); color code for atoms: grey $=\mathrm{C}$; blue $=\mathrm{N}$; red $=\mathrm{O}$; yellow $=\mathrm{Na}$; turquoise $=\mathrm{W}$.

Discussion of calculated sodium tungsten ATI 12. Compound 12 (Scheme S1) was calculated to be a stable isomer of $\mathbf{9}$ (Table S4). The tungsten atom of $\mathbf{1 2}$ is located in the chelating $N, N$ binding pocket of the ATI ligand. The sodium atom interacts with two pyridine ligands, with one carbonyl ligand, which is bridging between $\mathrm{W}$ and $\mathrm{Na}$, and with the ATI ligand backbone. The interaction between $\mathrm{Na}$ and the carbonyl ligand induces a proximity to the ligand backbone. Nevertheless, $\mathrm{N}-\mathrm{M}$ bond indices $(\mathrm{Na}-\mathrm{C} \approx 0.01)$ suggest weak interactions between $\mathrm{Na}$ and five carbon atoms of the ATI ligand backbone (C1, C2, C3, C6, $\mathrm{C} 7$ with $\mathrm{CNiPr}=\mathrm{C} 1$ ). Compared to compound 11, the Na-carbonyl interactions in $\mathbf{1 2}$ lead to a shift of the sodium atom across the ATI ligand backbone towards the nitrogen atoms.

Since one purpose of this work was to evaluate the interaction of metal atoms with the ATI ligand backbone in the absence of any chelating effects, compound $\mathbf{1 2}$ is not discussed in detail in the main text. However, its low Gibbs energy compared to $\mathbf{9}$ confirms that the ATI ligand backbone should be considered as a potential coordination site. 
a)

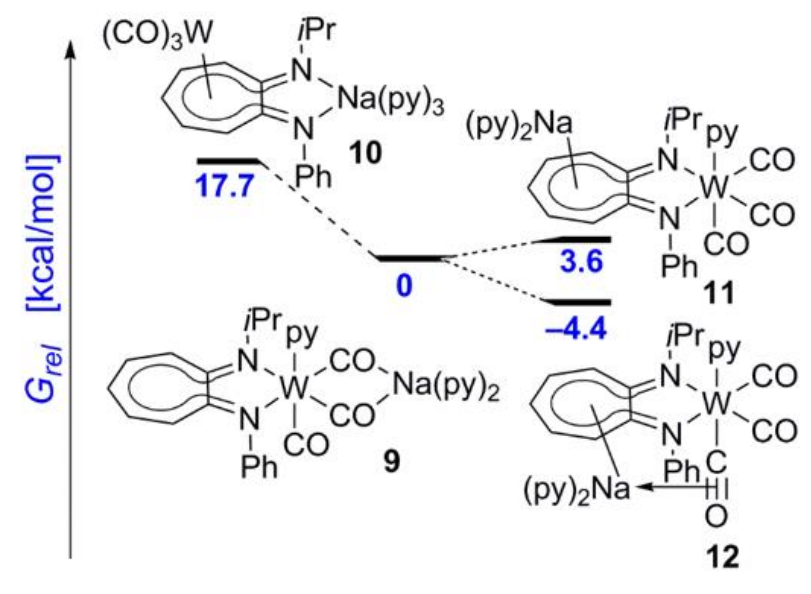

b)

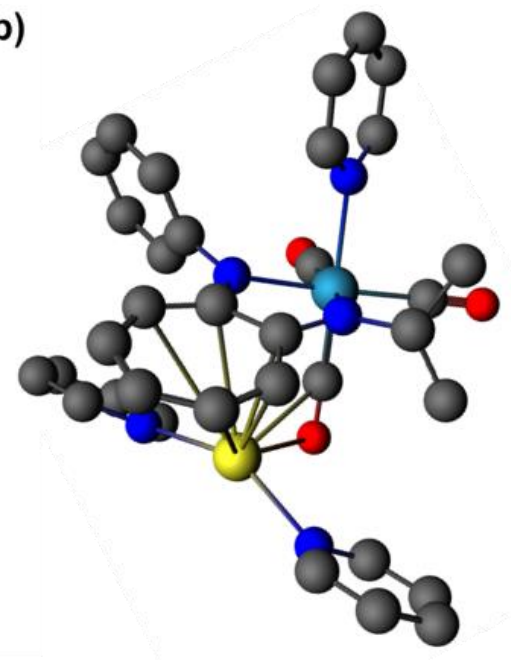

Scheme S1. a) Energy diagram of calculated isomers $\left[\mathrm{NaW}\left(\mathrm{ATI}^{\mathrm{Ph} / \mathrm{Pr}}\right)(\mathrm{CO})_{3}(\mathrm{py})_{3}\right](\mathbf{9 - 1 2})$; b) calculated structure of $\left[\mathrm{NaW}\left(\mathrm{ATI}^{\mathrm{Ph} / i \mathrm{Pr}}-1 \kappa^{5} C, 2 \kappa^{2} N\right)(\mathrm{CO}-2 \kappa C)_{2}\left(\mu_{2} \mathrm{CO}-1 \kappa C O, 2 \kappa C\right)(\right.$ py-

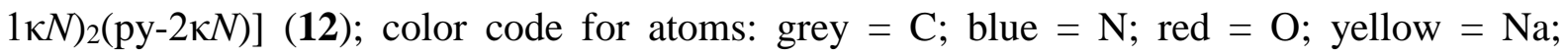
turquoise $=\mathrm{W}$.

Table S4. Relative enthalpies and free enthalpies of calculated tungsten ATI compounds.

\begin{tabular}{|c|c|c|}
\hline \multicolumn{3}{|c|}{$\left[\mathrm{NaW}\left(\mathrm{ATI}^{\mathrm{Ph} / i \mathrm{Pr}}\right)(\mathrm{CO})_{3}(\mathrm{py})_{3}\right]$} \\
\hline Compound & $\begin{array}{c}\Delta \boldsymbol{H}_{\text {rel }} \\
{\left[\mathrm{kcal} \cdot \mathrm{mol}^{-1}\right]}\end{array}$ & $\begin{array}{c}\Delta \boldsymbol{G}_{\boldsymbol{r} \boldsymbol{r l}} \\
{\left[\mathrm{kcal} \cdot \mathrm{mol}^{-1}\right]}\end{array}$ \\
\hline 9 & - & - \\
\hline 10 & 21.7 & 17.7 \\
\hline 11 & 7.5 & 3.6 \\
\hline 12 & -4.1 & -4.4 \\
\hline
\end{tabular}

Comparison of 7 (exp.) and 9 (calc.). The calculated compound 9 is a monomeric model for the experimentally observed compound 7. When comparing the bonding parameters of these compounds, one has to keep in mind that $\mathbf{7}$ forms a polymeric structure in the solid state. The coordination geometry around $\mathrm{W}$ is octahedral in both cases and $\mathrm{W}-\mathrm{N}$ bond lengths are well reproduced (Table S5). W-C bond lengths of the calculated structure show moderate deviations from those in $\mathbf{7}$, because the terminal carbonyl ligand is in trans position to the $\mathrm{NiPr}$ group in 9, whereas it is in trans position to the pyridine ligand in the experimentally observed case. Differences in $\mathrm{Na} 1-\mathrm{O}$ and $\mathrm{Na} 1-\mathrm{N}$ bond lengths are ascribed to the difference in the coordination number and geometry around $\mathrm{Nal}$ in $7(\mathrm{CN}=5$, distorted square pyramidal, isocarbonyl ligands in trans position) and $9(\mathrm{CN}=4$, distorted tetrahedral $)$. 
Table S5. Comparison of bond lengths involving W1 and Na1 of 7 (experimental, solid state, XRD) and 9 (calculated, gas phase); Atom labels according to Fig. 5 in main text.

\begin{tabular}{|c|c|c|}
\hline & $\begin{array}{c}\text { Compound } 7 \text { (experimental, XRD) } \\
{[\AA \AA} \\
\end{array}$ & $\begin{array}{c}\text { Compound } 9 \text { (calculated) } \\
{[\AA \AA]}\end{array}$ \\
\hline W1-N1 & $2.242(8)$ & 2.2561 \\
\hline W1-N2 & $2.167(7)$ & 2.2077 \\
\hline W1-N3 & $2.311(9)$ & 2.3298 \\
\hline W1-C22 & $1.913(10)$ & 1.9595 \\
\hline W1-C23 & $1.926(10)$ & 1.9408 \\
\hline W1-C24 & $1.930(9)$ & 1.9382 \\
\hline Na1-O & $2.350(8)-2.366(7)$ & $2.3865-2.5126$ \\
\hline $\mathbf{N a 1 - N}$ & $2.397(9)-2.475(12)$ & $2.4128-2.4384$ \\
\hline
\end{tabular}

\section{DFT calculations: sterically encumbered Rh ATI compounds}

Rhodium compounds with sterically encumbered monoanionic ATI ligands, $\left[\mathrm{ATI}^{t \mathrm{Bu} / \mathrm{BBu}}\right]^{-}$and neutral cod ligands (cod, $\mathrm{Me}_{2}-\mathrm{cod}$, and $\mathrm{Me}_{4}-\mathrm{cod}$ ) were considered ( $\mathrm{Me}_{2}-\mathrm{cod}=1,6-$ dimethylcycloocta-1,5-diene; $\mathrm{Me}_{4}$-cod $=$ 1,2,5,6-tetramethylcycloocta-1,5-diene). These calculations were performed with the Gaussian09 program package. ${ }^{1}$ All these structures were optimized using the $6-31 \mathrm{G}(\mathrm{d}, \mathrm{p})^{2}(\mathrm{H}, \mathrm{C}, \mathrm{N})$ or the LanL2DZ ${ }^{3}(\mathrm{Rh})$ basis set and the B3LYP functional. ${ }^{4,5}$ Frequency analyses of the reported structures showed no imaginary frequencies. For compound $\left[\mathrm{Rh}\left(\mathrm{ATI}^{\mathrm{IBu} / t \mathrm{Bu}}\right)(\mathrm{cod})\right](\mathbf{1 3})$, an isomer with a square planar coordination geometry around $\mathrm{Rh}(\mathbf{1 3}-\mathbf{s p})$ is energetically favored over the isomer with the rhodium center bound to the ligand backbone (13-pi) by $\Delta G_{r e l}=-3.1 \mathrm{kcal} \cdot \mathrm{mol}^{-1}$ (Table S6, Figure S12). ${ }^{6}$

The steric bulk around the olefinic carbon atoms of the cod ligand was increased stepwise. With two of the olefinic carbon atoms bearing methyl substituents, the energy gap between the two isomers of $\left[\mathrm{Rh}\left(\mathrm{ATI}^{\mathrm{tBu} / \mathrm{BBu}}\right)\left(\mathrm{Me}_{2}-\mathrm{cod}\right)\right]$ (14-sp and 14-pi) does not change significantly, the compound with the metal center in the $N, N$ binding pocket remaining lower in energy (Table S6, Figure S12). In 14-sp, the steric bulk of the ligands induces a tilt of the ATI ligand such that the ligand backbone and the metal center are not in plane. An additional structure was found as a minimum on the potential energy surface, 14-tet. It shows a tetrahedral coordination geometry around $\mathrm{Rh}$ and is slightly higher in energy than 14-pi (Table S6).

When two more methyl groups were added to the cod ligand (to give $\left[R h\left(\mathrm{ATI}^{t \mathrm{Bu} / \mathrm{Bu}}\right)\left(\mathrm{Me}_{4}-\right.\right.$ cod)]), the structure with the metal atom binding to the ATI ligand backbone, (15-pi), becomes marginally lower in energy than isomeric structures with the metal atom in the $N, N$ binding pocket (Table S6, Figure S12). It has to be pointed out that when starting a geometry optimization with a square planar coordination geometry around $\mathrm{Rh}$, a converged structure with a distorted tetrahedral coordination geometry was obtained, 15-tet. An additional structure was found that represents a minimum on the potential energy surface and shows a tetrahedral coordination geometry around Rh, 15-tet'.

Thus, calculations suggest that interactions of an anionic ATI ligand with a cationic metal center via its $\mathrm{C}_{7}$ backbone or its $N, N$ binding pocket become similar in energy, if the steric bulk around the $\mathrm{N}^{\mathrm{ATI}}$ atoms and the additional neutral ligand bound to the metal center are high. The calculated relative energies are summarized in Table S6. 
Table S6. Relative enthalpies and free enthalpies of calculated Rh ATI compounds. Values are given in $\mathrm{kcal} \cdot \mathrm{mol}^{-1}$ (B3LYP; 6-31G(d,p) (H, C, N), LanL2DZ (Rh)).

\begin{tabular}{|c|c|c|c|c|c|c|c|c|}
\hline \multicolumn{3}{|c|}{$\left[\mathrm{Rh}\left(\mathrm{ATI}^{t \mathrm{Bu} / t \mathrm{Bu}}\right)(\operatorname{cod})\right]$} & \multicolumn{3}{|c|}{$\left[\mathrm{Rh}\left(\mathrm{ATI}^{t \mathrm{Bu} / t \mathrm{Bu}}\right)\left(\mathrm{Me}_{2}-\mathrm{cod}\right)\right]$} & \multicolumn{3}{|c|}{$\left[\mathrm{Rh}\left(\mathrm{ATI}^{t \mathrm{Bu} / t \mathrm{Bu}}\right)\left(\mathrm{Me}_{4}-\mathrm{cod}\right)\right]$} \\
\hline Cpd & $\Delta H_{\text {rel }}$ & $\Delta G_{r e l}$ & Cpd & $\Delta H_{r e l}$ & $\Delta G_{r e l}$ & Cpd & $\Delta H_{r e l}$ & $\Delta G_{r e l}$ \\
\hline 13-sp & - & - & 14-sp & - & - & 15-pi & - & - \\
\hline 13-pi & +5.8 & +3.1 & 14-pi & +5.8 & +3.5 & 15-tet & +5.4 & +7.5 \\
\hline & & & 14-tet & +6.1 & +5.1 & 15-tet' & +4.4 & +7.0 \\
\hline
\end{tabular}

a)

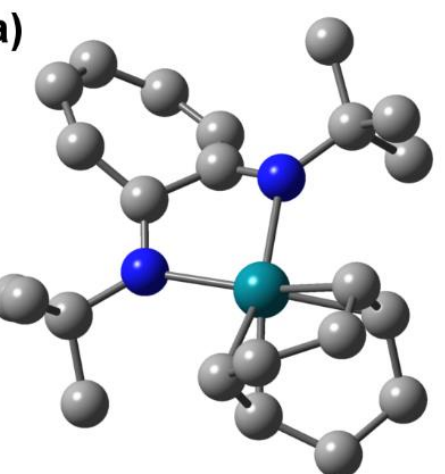

13-sp

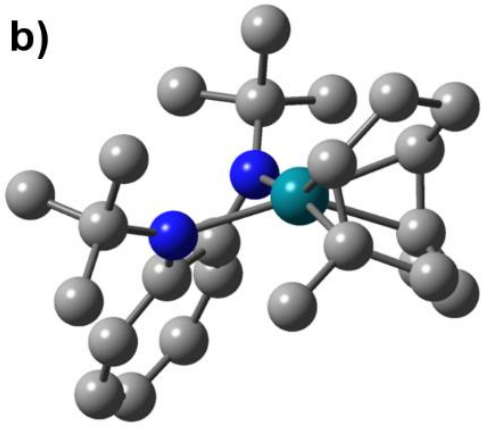

14-sp

c)

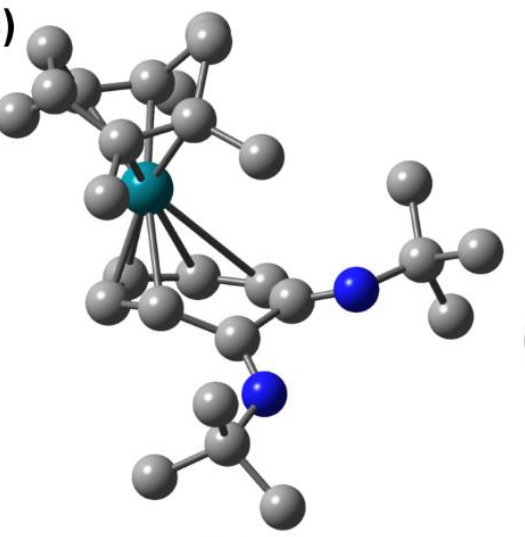

15-pi

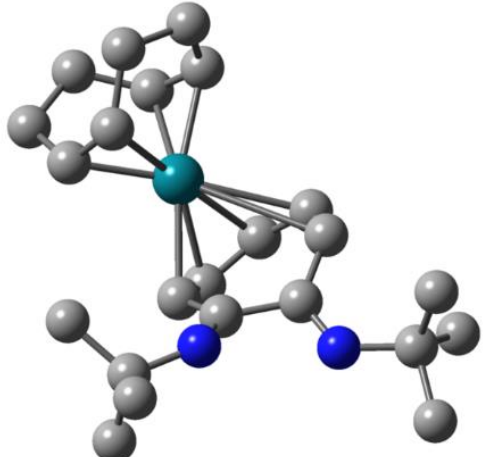

13-pi

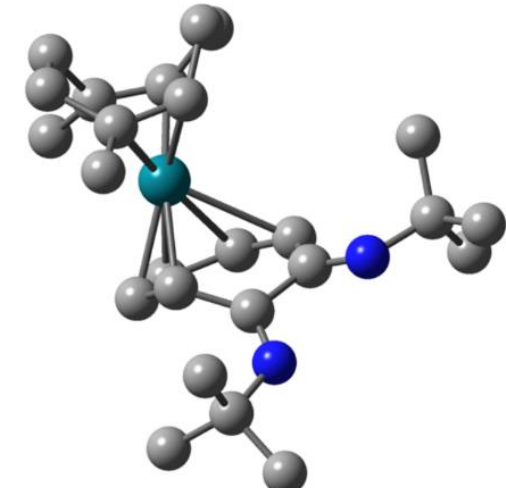

14-pi

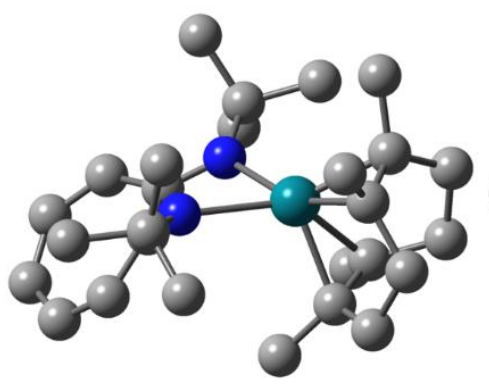

15-tet

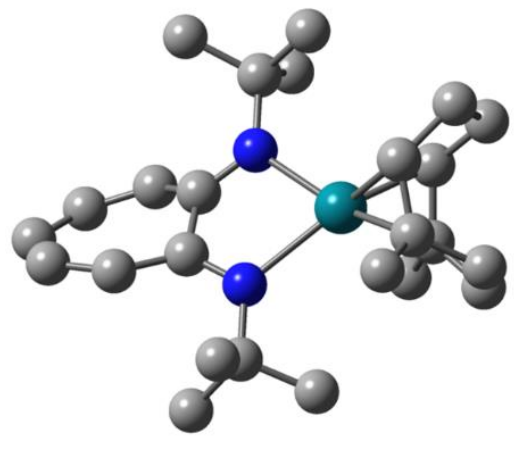

14-tet

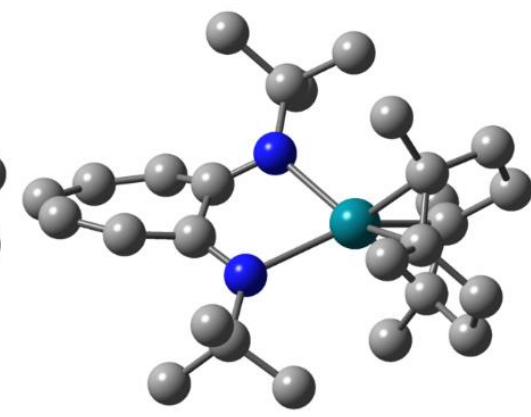

15-tet'

Figure S12. Structures of calculated compounds a) $\left.\left[\operatorname{Rh}\left(\mathrm{ATI}^{I_{\mathrm{Bu}} / \mathrm{Bu} u}\right)(\operatorname{cod})\right](\mathbf{1 3}) ; \mathrm{b}\right)$ $\left.\left[\mathrm{Rh}\left(\mathrm{ATI}^{t \mathrm{Bu} / \mathrm{Bu}}\right)\left(\mathrm{Me}_{2}-\mathrm{cod}\right)\right](\mathbf{1 4}) ; \mathrm{c}\right)\left[\mathrm{Rh}\left(\mathrm{ATI}^{t \mathrm{Bu} / t \mathrm{Bu}}\right)\left(\mathrm{Me}_{4}-\mathrm{cod}\right)\right]$ (15) with different coordination modes or geometries. Color code for atoms: grey $=\mathrm{C}$; blue $=\mathrm{N}$; turquoise $=\mathrm{Rh}$. 
Total bonding energies (TBE, $\mathrm{kcal} / \mathrm{mol})$, entropies $\left(S^{\circ}, \mathrm{cal} / \mathrm{mol}\right)$, and cartesian coordinates $(\AA)$ of optimized structures.

\begin{tabular}{|c|c|c|c|}
\hline \multicolumn{4}{|c|}{$\left[\mathrm{Na}\left(\mathrm{ATI} \mathrm{T}^{\mathrm{Ph} / \mathrm{Pr}}\right)(\mathrm{thf})\right]$} \\
\hline \multicolumn{4}{|c|}{$\mathrm{TBE}=-6778.74$} \\
\hline \multicolumn{4}{|c|}{$S^{0}=168.596$} \\
\hline $\mathrm{N}$ & -2.273079 & 0.983628 & 0.918398 \\
\hline $\mathrm{N}$ & -0.398045 & -0.399084 & -0.325290 \\
\hline $\mathrm{C}$ & -1.628049 & -0.898287 & -0.425509 \\
\hline $\mathrm{C}$ & -2.698100 & -0.113709 & 0.297883 \\
\hline $\mathrm{C}$ & -3.205702 & 1.827531 & 1.657949 \\
\hline $\mathrm{H}$ & -4.179612 & 1.911355 & 1.138578 \\
\hline $\mathrm{C}$ & -4.761481 & -1.590614 & -0.248527 \\
\hline $\mathrm{H}$ & -5.830397 & -1.601788 & -0.016739 \\
\hline $\mathrm{C}$ & 0.728111 & -1.042067 & -0.840269 \\
\hline $\mathrm{C}$ & -2.998626 & -2.822305 & -1.476797 \\
\hline $\mathrm{H}$ & -2.822312 & -3.688206 & -2.121100 \\
\hline $\mathrm{C}$ & -4.067711 & -0.530263 & 0.322668 \\
\hline $\mathrm{H}$ & -4.711584 & 0.115891 & 0.917084 \\
\hline C & 1.340285 & -2.109528 & -0.147501 \\
\hline $\mathrm{H}$ & 0.835862 & -2.511800 & 0.731387 \\
\hline $\mathrm{C}$ & 2.568042 & -2.621624 & -0.564697 \\
\hline $\mathrm{H}$ & 3.026981 & -3.438563 & -0.006264 \\
\hline $\mathrm{C}$ & -4.315928 & -2.634592 & -1.061450 \\
\hline $\mathrm{H}$ & -5.058235 & -3.359627 & -1.397738 \\
\hline $\mathrm{C}$ & -1.861388 & -2.074084 & -1.196961 \\
\hline $\mathrm{H}$ & -0.952382 & -2.455722 & -1.665375 \\
\hline C & 1.377139 & -0.538476 & -1.986097 \\
\hline $\mathrm{H}$ & 0.901914 & 0.275687 & -2.534365 \\
\hline $\mathrm{C}$ & -3.452180 & 1.270442 & 3.071650 \\
\hline $\mathrm{H}$ & -3.852499 & 0.250152 & 3.028956 \\
\hline $\mathrm{H}$ & -4.155905 & 1.900399 & 3.634034 \\
\hline $\mathrm{H}$ & -2.502267 & 1.233783 & 3.625664 \\
\hline $\mathrm{C}$ & -2.635405 & 3.250068 & 1.739856 \\
\hline $\mathrm{H}$ & -1.692978 & 3.262009 & 2.314179 \\
\hline $\mathrm{H}$ & -3.331063 & 3.928135 & 2.252988 \\
\hline $\mathrm{H}$ & -2.442120 & 3.651395 & 0.735647 \\
\hline C & 3.214364 & -2.094605 & -1.688857 \\
\hline $\mathrm{H}$ & 4.175385 & -2.494610 & -2.010670 \\
\hline C & 2.604747 & -1.056855 & -2.399650 \\
\hline $\mathrm{H}$ & 3.090434 & -0.645471 & -3.285625 \\
\hline $\mathrm{O}$ & 2.253921 & 1.897117 & 1.225655 \\
\hline $\mathrm{C}$ & 3.246940 & 1.076066 & 0.525752 \\
\hline $\mathrm{H}$ & 3.316109 & 1.425729 & -0.515291 \\
\hline $\mathrm{H}$ & 2.892795 & 0.038680 & 0.519501 \\
\hline $\mathrm{C}$ & 2.936384 & 2.836013 & 2.103092 \\
\hline $\mathrm{H}$ & 2.760120 & 2.530521 & 3.147604 \\
\hline $\mathrm{H}$ & 2.503985 & 3.834125 & 1.947295 \\
\hline $\mathrm{C}$ & 4.550505 & 1.289785 & 1.284182 \\
\hline $\mathrm{H}$ & 4.603879 & 0.618286 & 2.152521 \\
\hline $\mathrm{H}$ & 5.429847 & 1.109322 & 0.655328 \\
\hline $\mathrm{C}$ & 4.418265 & 2.751426 & 1.739468 \\
\hline $\mathrm{H}$ & 4.648417 & 3.432171 & 0.908261 \\
\hline
\end{tabular}

\author{
$\begin{array}{llll}\mathrm{H} & 5.068741 & 3.007633 & 2.583963\end{array}$ \\ $\mathrm{Na} \quad-0.009110 \quad 1.462744 \quad 0.915172$
}

$\left[\mathrm{Na}\left(\mathrm{ATI} \mathrm{I}^{\mathrm{Ph} / \mathrm{Pr}}\right)(\mathrm{thf})_{2}\right]$

$\mathrm{TBE}=-8442.88$

$S^{0}=201.848$

$\begin{array}{llll}\mathrm{O} & 1.909452 & 0.159710 & 2.362424\end{array}$

$\begin{array}{llll}\mathrm{N} & -1.765638 & 0.987680 & 1.055993\end{array}$

$\begin{array}{llll}\mathrm{N} & -0.045422 & -0.480448 & -0.360956\end{array}$

$\begin{array}{llll}\text { C } & -1.343977 & -0.697002 & -0.597297\end{array}$

$\begin{array}{llll}\text { C } & -2.287621 & -0.002304 & 0.350121\end{array}$

C $\quad-2.596391 \quad 1.743332 \quad 1.988265$

$\mathrm{H} \quad-3.652310 \quad 1.774353 \quad 1.659425$

$\begin{array}{llll}\text { C } & -4.469232 & -1.262577 & -0.244958\end{array}$

$\begin{array}{llll}\mathrm{H} & -5.467872 & -1.414319 & 0.174667\end{array}$

$\begin{array}{llll}\text { C } & 0.960045 & -1.274996 & -0.890377\end{array}$

$\begin{array}{llll}\text { C } & -3.021416 & -1.884366 & -2.158383\end{array}$

$\begin{array}{llll}\mathrm{H} & -3.018992 & -2.382545 & -3.131825\end{array}$

$\begin{array}{llll}\text { C } & -3.633185 & -0.476106 & 0.533880\end{array}$

$\begin{array}{llll}\mathrm{H} & -4.098585 & -0.133681 & 1.457208\end{array}$

$\begin{array}{lllll}\text { C } & 0.920630 & -2.692056 & -0.876131\end{array}$

$\begin{array}{llll}\mathrm{H} & 0.014354 & -3.184387 & -0.524436\end{array}$

$\begin{array}{llll}\text { C } & 2.018051 & -3.445881 & -1.281493\end{array}$

$\begin{array}{lllll}\mathrm{H} & 1.959965 & -4.534716 & -1.246945\end{array}$

$\begin{array}{llll}\text { C } & -4.243711 & -1.857269 & -1.491809\end{array}$

$\begin{array}{llll}\mathrm{H} & -5.087495 & -2.359563 & -1.966672\end{array}$

$\begin{array}{llll}\text { C } & -1.775783 & -1.419043 & -1.742908\end{array}$

$\begin{array}{llll}\mathrm{H} & -0.957352 & -1.653716 & -2.427271\end{array}$

$\begin{array}{llll}\text { C } & 2.158767 & -0.668363 & -1.333416\end{array}$

$\begin{array}{llll}\mathrm{H} & 2.211374 & 0.420646 & -1.348809\end{array}$

$\begin{array}{llll}\text { C } & -2.536793 & 1.107229 & 3.387926\end{array}$

$\begin{array}{llll}\mathrm{H} & -2.882818 & 0.066033 & 3.364856\end{array}$

$\begin{array}{llll}\mathrm{H} & -3.151778 & 1.665397 & 4.107882\end{array}$

$\begin{array}{llll}\mathrm{H} & -1.498103 & 1.105460 & 3.750580\end{array}$

$\begin{array}{llll}\text { C } & -2.099922 & 3.194462 & 2.035988\end{array}$

$\begin{array}{llll}\mathrm{H} & -1.074692 & 3.247202 & 2.436720\end{array}$

$\begin{array}{llll}\mathrm{H} & -2.737334 & 3.806975 & 2.688268\end{array}$

$\begin{array}{llll}\mathrm{H} & -2.098852 & 3.640081 & 1.032851\end{array}$

$\begin{array}{llll}\text { C } & 3.262245 & -0.283637 & 2.062747\end{array}$

$\begin{array}{llll}\mathrm{H} & 3.932675 & 0.080271 & 2.859016\end{array}$

$\begin{array}{llll}\mathrm{H} & 3.567430 & 0.157218 & 1.103447\end{array}$

$\begin{array}{llll}\text { C } & 1.107065 & -0.989534 & 2.785326\end{array}$

$\begin{array}{llll}\mathrm{H} & 0.401150 & -1.228423 & 1.974759\end{array}$

$\begin{array}{llll}\mathrm{H} & 0.543871 & -0.691858 & 3.679577\end{array}$

$\begin{array}{llll}\text { C } & 3.206256 & -1.807755 & 2.013772\end{array}$

$\begin{array}{llll}\mathrm{H} & 2.911390 & -2.142093 & 1.011913\end{array}$

$\begin{array}{llll}\mathrm{H} & 4.169265 & -2.268126 & 2.264863\end{array}$

$\begin{array}{llll}\text { C } & 3.194826 & -2.825441 & -1.720215\end{array}$

$\begin{array}{llll}\mathrm{H} & 4.051040 & -3.420712 & -2.035043\end{array}$

$\begin{array}{llll}\text { C } & 3.252810 & -1.429107 & -1.741415\end{array}$ 


$\begin{array}{lccc}\mathrm{H} & 4.161242 & -0.926800 & -2.077111 \\ \mathrm{C} & 2.099413 & -2.122632 & 3.031023 \\ \mathrm{H} & 2.491193 & -2.081598 & 4.057399 \\ \mathrm{H} & 1.644351 & -3.107158 & 2.872184 \\ \mathrm{O} & 0.730105 & 2.895867 & -0.894170 \\ \mathrm{C} & -0.471284 & 2.734248 & -1.693647 \\ \mathrm{H} & -0.860528 & 3.730882 & -1.969542 \\ \mathrm{H} & -1.205843 & 2.209591 & -1.071095 \\ \mathrm{C} & 1.792823 & 3.193500 & -1.827990 \\ \mathrm{H} & 2.727835 & 2.821040 & -1.388937 \\ \mathrm{H} & 1.875178 & 4.286783 & -1.945535 \\ \mathrm{C} & -0.002377 & 1.948131 & -2.913564 \\ \mathrm{H} & 0.038075 & 0.883462 & -2.656744 \\ \mathrm{H} & -0.667878 & 2.072398 & -3.775553 \\ \mathrm{C} & 1.418098 & 2.508181 & -3.165913 \\ \mathrm{H} & 1.423349 & 3.239349 & -3.983460 \\ \mathrm{H} & 2.121341 & 1.709072 & -3.426864 \\ \mathrm{Na} & 0.540373 & 1.396624 & 0.919971\end{array}$

\begin{tabular}{|c|c|c|c|}
\hline \multicolumn{4}{|c|}{$\left[\mathrm{Na}_{2}\left(\mathrm{ATI}^{\mathrm{Ph} / \mathrm{Pr}}\right)_{2}(\mathrm{thf})_{3}\right](8)$} \\
\hline \multicolumn{4}{|c|}{$\mathrm{TBE}=-15248.09$} \\
\hline \multicolumn{4}{|c|}{$S^{0}=316.887$} \\
\hline $\mathrm{Na}$ & -3.715221 & 1.137112 & 0.331054 \\
\hline $\mathrm{Na}$ & 1.876413 & -0.733470 & -0.707988 \\
\hline $\mathrm{N}$ & 3.689473 & 0.657202 & -0.328459 \\
\hline $\mathrm{O}$ & 0.636675 & -0.306161 & 1.344509 \\
\hline C & 2.923656 & 2.981970 & -0.394553 \\
\hline $\mathrm{H}$ & 2.474339 & 2.858256 & 0.589599 \\
\hline $\mathrm{N}$ & 3.616212 & -1.900344 & 0.331632 \\
\hline $\mathrm{C}$ & 4.727201 & 0.223407 & 0.382013 \\
\hline $\mathrm{C}$ & 3.672455 & 1.916244 & -0.931830 \\
\hline $\mathrm{C}$ & 4.131038 & 3.307532 & -2.883826 \\
\hline $\mathrm{H}$ & 4.611027 & 3.429677 & -3.855859 \\
\hline $\mathrm{C}$ & 4.297114 & 2.115604 & -2.181885 \\
\hline $\mathrm{H}$ & 4.890744 & 1.300682 & -2.596472 \\
\hline $\mathrm{C}$ & 4.668595 & -1.230697 & 0.790559 \\
\hline $\mathrm{C}$ & 7.447118 & -0.119004 & 2.156955 \\
\hline $\mathrm{H}$ & 8.383666 & 0.007944 & 2.701646 \\
\hline C & 5.675515 & -1.840162 & 1.607205 \\
\hline $\mathrm{H}$ & 5.491609 & -2.893512 & 1.813311 \\
\hline $\mathrm{C}$ & 1.430005 & -0.567425 & 2.536097 \\
\hline $\mathrm{H}$ & 2.211257 & -1.284987 & 2.260859 \\
\hline $\mathrm{H}$ & 0.772926 & -1.001274 & 3.310146 \\
\hline $\mathrm{C}$ & 3.489710 & -3.336242 & 0.565876 \\
\hline $\mathrm{H}$ & 3.790632 & -3.616375 & 1.594927 \\
\hline $\mathrm{C}$ & 5.795855 & 1.113308 & 0.701747 \\
\hline $\mathrm{H}$ & 5.655965 & 2.107158 & 0.272671 \\
\hline $\mathrm{C}$ & 6.953323 & 0.982734 & 1.459855 \\
\hline $\mathrm{H}$ & 7.559483 & 1.891506 & 1.517613 \\
\hline C & 6.842678 & -1.376774 & 2.202886 \\
\hline $\mathrm{H}$ & 7.374333 & -2.130373 & 2.791612 \\
\hline $\mathrm{C}$ & 4.363244 & -4.123500 & -0.426558 \\
\hline $\mathrm{H}$ & 4.043266 & -3.898642 & -1.454030 \\
\hline
\end{tabular}

\begin{tabular}{|c|c|c|c|}
\hline & 4.276834 & -5.207135 & \\
\hline & 5.418211 & -3.837743 & -0.333404 \\
\hline & 2.756273 & 4.172603 & -1.103095 \\
\hline & 2.160078 & 4.976713 & -0.669450 \\
\hline & 0.279776 & 1.105360 & 1.314648 \\
\hline & -0.804972 & 1.189588 & 1.182290 \\
\hline & 0.758069 & 1.558308 & 0.434700 \\
\hline & 1.980137 & 960 & 130 \\
\hline & 2.857484 & 1.03 & 943 \\
\hline & 2.268375 & 0.82 & 4.01 \\
\hline & 3.346750 & & -2.3 \\
\hline & 3.208645 & 5.26 & -2.91 \\
\hline & 2.018076 & -3.743842 & 0.42 \\
\hline & 1.389542 & -3.213240 & 8045 \\
\hline & 1.888527 & -4.822268 & 0.58 \\
\hline & 1.642748 & -3.521534 & -0.591087 \\
\hline & 0.803201 & 1.716059 & 000 \\
\hline & 0.036023 & 1.670133 & 3.35 \\
\hline & 1.093965 & 2.766273 & 2.48 \\
\hline & -2.346801 & -0.5 & -0.5 \\
\hline & -5.503857 & 0.85 & 5264 \\
\hline & -3.861032 & -2.3 & 069 \\
\hline & -4.683629 & -1.75 & -0.3 \\
\hline & -2.032892 & 636 & -1.0 \\
\hline & -1.429674 & -0.243020 & -1.4 \\
\hline C & -2.549342 & -1.854014 & -0.08 \\
\hline C & -1.754497 & 2204 & 0.9 \\
\hline $\mathrm{H}$ & -0.921430 & -4.50 & 1.3 \\
\hline & -1.502255 & -2.6 & 0.4 \\
\hline $\mathrm{H}$ & -0.495112 & -2.24 & 0.418367 \\
\hline & -1.155332 & 1.23 & -1.62 \\
\hline & 1.062696 & -0.19 & -3.5 \\
\hline $\mathrm{H}$ & 1.863344 & -0.42 & \\
\hline & & & \\
\hline & 0.283580 & 2.74 & -2.03 \\
\hline & -4.954472 & 0.937985 & -2.51 \\
\hline & -3.930195 & 1.320149 & -2.4 \\
\hline $\mathrm{H}$ & -5.560585 & 1.621818 & -3.13 \\
\hline C & -1.879558 & 3.497874 & -1.182408 \\
\hline $\mathrm{H}$ & -1.405418 & 3.770887 & -2.1 \\
\hline & -0.830160 & -1.251507 & -2.267515 \\
\hline $\mathrm{H}$ & -1.288062 & -2.232261 & -2.127444 \\
\hline $\mathrm{C}$ & 0.203983 & -1.236160 & -3.203688 \\
\hline $\mathrm{H}$ & 0.388890 & -2.199035 & -3.688294 \\
\hline & 0.991702 & 1.114040 & -3.077401 \\
\hline & 1.799883 & 1.781721 & -3.383266 \\
\hline & -1.014382 & 4.054790 & -0.038312 \\
\hline & -1.457531 & 3.787709 & 0.932356 \\
\hline & -0.945401 & 5.149808 & -0.095001 \\
\hline $\mathrm{H}$ & 0.002543 & 3.646209 & -0.069069 \\
\hline $\mathrm{C}$ & -4.110214 & -3.626373 & 0.551620 \\
\hline F & -5.135389 & -3.996019 & 0.600962 \\
\hline & -6.632047 & -0.010089 & -1.215455 \\
\hline & -7.557901 & 0.570359 & -1.35743 \\
\hline
\end{tabular}




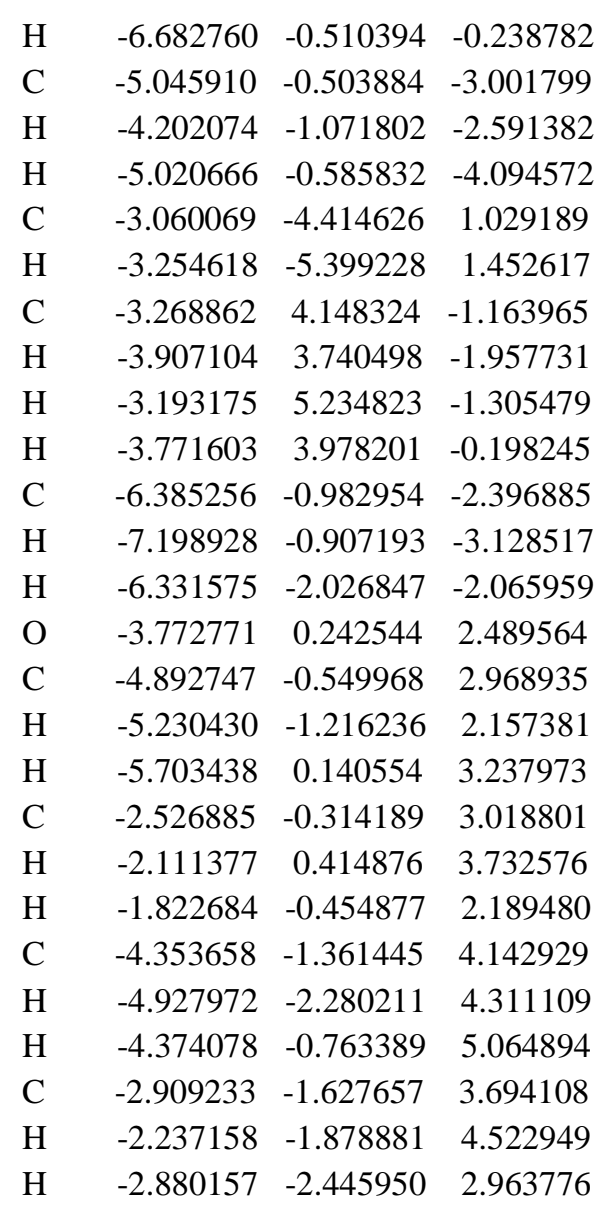

\section{THF}

$\mathrm{TBE}=-1643.98$

$S^{0}=72.001$

$\begin{array}{llll}\text { O } & 0.000531 & -1.259464 & 0.000630\end{array}$

$\begin{array}{llll}\text { C } & -1.170375 & -0.425564 & 0.135391\end{array}$

$\begin{array}{llll}\mathrm{H} & -1.530126 & -0.471643 & 1.178993\end{array}$

$\begin{array}{llll}\mathrm{H} & -1.957655 & -0.822018 & -0.521771\end{array}$

$\begin{array}{llll}\text { C } & 1.170482 & -0.424655 & -0.136186\end{array}$

$\begin{array}{llll}\mathrm{H} & 1.528515 & -0.470051 & -1.180432\end{array}$

$\begin{array}{llll}\mathrm{H} & 1.959138 & -0.820719 & 0.519516\end{array}$

$\begin{array}{lllll}\text { C } & -0.732267 & 0.995404 & -0.232563\end{array}$

$\begin{array}{llll}\mathrm{H} & -0.786880 & 1.144542 & -1.320353\end{array}$

$\begin{array}{llll}\mathrm{H} & -1.345346 & 1.765467 & 0.251686\end{array}$

$\begin{array}{llll}\text { C } & 0.731719 & 0.995730 & 0.232860\end{array}$

$\begin{array}{llll}\mathrm{H} & 0.786328 & 1.144013 & 1.320766\end{array}$

$\begin{array}{llll}\mathrm{H} & 1.344320 & 1.766522 & -0.250885\end{array}$

\section{[ $\left.\mathrm{Na}\left(\mathrm{C}_{5} \mathrm{H}_{5}\right)(\text { thf })_{3}\right]$}

TBE $=-6434.49$

$$
\begin{array}{lccc}
\multicolumn{4}{l}{S^{0}=178.561} \\
\mathrm{Na} & 5.877086 & 9.172783 & 2.723481 \\
\mathrm{O} & 3.761858 & 9.189892 & 1.647573 \\
\mathrm{O} & 6.276501 & 11.098452 & 1.362533 \\
\mathrm{O} & 6.663427 & 7.256022 & 1.451315 \\
\mathrm{C} & 3.429942 & 9.639956 & 0.319702
\end{array}
$$

\begin{tabular}{cccc}
$\mathrm{C}$ & 2.546236 & 8.633308 & 2.182516 \\
$\mathrm{C}$ & 7.642777 & 11.586416 & 1.507393 \\
$\mathrm{C}$ & 5.351136 & 12.143329 & 1.770081 \\
$\mathrm{C}$ & 7.382316 & 6.307351 & 2.284149 \\
$\mathrm{C}$ & 5.620040 & 6.497577 & 0.811067 \\
$\mathrm{C}$ & 2.413743 & 8.613311 & -0.233642 \\
$\mathrm{C}$ & 1.964338 & 7.818275 & 1.023256 \\
$\mathrm{C}$ & 7.534225 & 13.075145 & 1.843392 \\
$\mathrm{C}$ & 6.181130 & 13.142299 & 2.568443 \\
$\mathrm{C}$ & 6.352420 & 5.236336 & 2.717975 \\
$\mathrm{C}$ & 5.075538 & 5.604561 & 1.924888 \\
$\mathrm{H}$ & 2.982495 & 10.647133 & 0.381098 \\
$\mathrm{H}$ & 4.367228 & 9.710993 & -0.243572 \\
$\mathrm{H}$ & 2.816128 & 8.054554 & 3.072754 \\
$\mathrm{H}$ & 1.866877 & 9.450804 & 2.484660 \\
$\mathrm{H}$ & 8.121853 & 11.030324 & 2.329505 \\
$\mathrm{H}$ & 8.179308 & 11.384044 & 0.570701 \\
$\mathrm{H}$ & 4.551969 & 11.671923 & 2.357629 \\
$\mathrm{H}$ & 4.915793 & 12.603976 & 0.866237 \\
$\mathrm{H}$ & 8.200270 & 5.864709 & 1.692347 \\
$\mathrm{H}$ & 7.798634 & 6.874047 & 3.124823 \\
$\mathrm{H}$ & 4.892173 & 7.210605 & 0.410057 \\
$\mathrm{H}$ & 6.042409 & 5.899563 & -0.017561 \\
$\mathrm{H}$ & 1.572480 & 9.120529 & -0.720719 \\
$\mathrm{H}$ & 2.874626 & 7.952256 & -0.977001 \\
$\mathrm{H}$ & 0.875499 & 7.711050 & 1.093156 \\
$\mathrm{H}$ & 2.403250 & 6.812747 & 1.020482 \\
$\mathrm{H}$ & 8.372025 & 13.420905 & 2.460337 \\
$\mathrm{H}$ & 7.509901 & 13.679561 & 0.925542 \\
$\mathrm{H}$ & 6.282372 & 12.790160 & 3.603025 \\
$\mathrm{H}$ & 5.741536 & 14.147097 & 2.575226 \\
$\mathrm{H}$ & 6.174437 & 5.269408 & 3.797710 \\
$\mathrm{H}$ & 6.705580 & 4.230356 & 2.459745 \\
$\mathrm{H}$ & 4.392477 & 6.188547 & 2.556376 \\
$\mathrm{H}$ & 8.140541 & 8.403001 & 5.051074 \\
$\mathrm{H}$ & 4.535674 & 4.728112 & 1.545970 \\
$\mathrm{C}$ & 4.858834 & 9.067182 & 5.156040 \\
$\mathrm{C}$ & 5.425799 & 10.362394 & 5.031920 \\
$\mathrm{C}$ & 5.917606 & 8.121802 & 5.150131 \\
$\mathrm{C}$ & 7.140655 & 8.833055 & 5.022260 \\
$\mathrm{H}$ & 6.835340 & 10.218143 & 4.950662 \\
$\mathrm{H}$ & 7.813148 & 7.047185 & 5.287839 \\
\hline & 4.875198 & 11.027479 & 4.903355 \\
$\mathrm{H}$ & 3.802099 & 8.843558 & 5.049875 \\
& & & \\
$\mathrm{H}$ & & &
\end{tabular}

\section{$\left[\mathrm{Na}\left(\mathrm{C}_{5} \mathrm{Me}_{5}\right)(\mathrm{thf})_{3}\right]$}

$\mathrm{TBE}=-8330.50$

\begin{tabular}{lccc}
\multicolumn{4}{l}{$S^{0}=213.271$} \\
$\mathrm{Na}$ & 5.408660 & 8.916688 & 2.989274 \\
$\mathrm{O}$ & 3.471829 & 8.753201 & 1.646991 \\
$\mathrm{O}$ & 6.413132 & 10.401717 & 1.390630 \\
$\mathrm{O}$ & 6.158881 & 6.964591 & 1.870439
\end{tabular} 


\begin{tabular}{|c|c|c|c|}
\hline C & 2.461365 & 9.795998 & 1.709205 \\
\hline $\mathrm{C}$ & 2.858717 & 7.468545 & 1.950455 \\
\hline $\mathrm{C}$ & 7.816207 & 10.737732 & 1.457120 \\
\hline . & 5.634728 & 11.633259 & 1.409818 \\
\hline C & 7.297728 & 6.331552 & 2.493961 \\
\hline C & 6.487906 & 7.173255 & 0.470980 \\
\hline C & 1.206447 & 9.140905 & 2.284414 \\
\hline $\mathrm{C}$ & 1.354638 & 7.686578 & 1.812680 \\
\hline C & 7.920723 & 12.116879 & 0.816129 \\
\hline C & 6.638484 & 12.795013 & 1.328139 \\
\hline $\mathrm{C}$ & 8.498140 & 6.992973 & 1.826711 \\
\hline $\mathrm{C}$ & 8.030214 & 7.092758 & 0.361030 \\
\hline $\mathrm{H}$ & 2.840154 & 10.612992 & 2.337147 \\
\hline $\mathrm{H}$ & 2.294026 & 10.175219 & 0.687631 \\
\hline $\mathrm{H}$ & 3.275478 & 6.728878 & 1.255796 \\
\hline $\mathrm{H}$ & 3.126483 & 7.179651 & 2.980061 \\
\hline $\mathrm{H}$ & 8.146711 & 10.752536 & 2.509828 \\
\hline $\mathrm{H}$ & 8.365798 & 9.954083 & 0.922058 \\
\hline $\mathrm{H}$ & 5.045175 & 11.666426 & 2.338630 \\
\hline $\mathrm{H}$ & 4.950307 & 11.595472 & 0.550600 \\
\hline $\mathrm{H}$ & 7.218945 & 6.511468 & 3.571236 \\
\hline $\mathrm{H}$ & 7.277144 & 5.243249 & 2.302431 \\
\hline $\mathrm{H}$ & 5.995925 & 6.395358 & -0.134247 \\
\hline $\mathrm{H}$ & 6.084754 & 8.154404 & 0.191770 \\
\hline $\mathrm{H}$ & 1.227509 & 9.186150 & 3.381400 \\
\hline $\mathrm{H}$ & 0.284746 & 9.622046 & 1.936777 \\
\hline $\mathrm{H}$ & 0.774181 & 6.977559 & 2.414767 \\
\hline $\mathrm{H}$ & 1.044071 & 7.587091 & 0.762835 \\
\hline $\mathrm{H}$ & 8.832702 & 12.651451 & 1.107863 \\
\hline $\mathrm{H}$ & 7.905033 & 12.025448 & -0.278850 \\
\hline $\mathrm{H}$ & 6.809228 & 13.215964 & 2.326196 \\
\hline $\mathrm{H}$ & 6.288479 & 13.599881 & 0.671684 \\
\hline $\mathrm{H}$ & 9.425825 & 6.419832 & 1.941886 \\
\hline $\mathrm{H}$ & 8.651908 & 7.987553 & 2.266754 \\
\hline $\mathrm{H}$ & 8.330398 & 6.196768 & -0.197208 \\
\hline $\mathrm{H}$ & 8.450317 & 7.961053 & -0.160126 \\
\hline $\mathrm{C}$ & 8.158652 & 9.113180 & 5.324726 \\
\hline $\mathrm{H}$ & 8.502610 & 8.109576 & 5.029761 \\
\hline $\mathrm{H}$ & 8.670389 & 9.830559 & 4.663589 \\
\hline $\mathrm{H}$ & 8.553511 & 9.293446 & 6.340094 \\
\hline $\mathrm{C}$ & 5.991268 & 6.802135 & 5.901007 \\
\hline $\mathrm{H}$ & 5.450224 & 6.057218 & 5.292379 \\
\hline $\mathrm{H}$ & 7.062343 & 6.563198 & 5.832530 \\
\hline $\mathrm{H}$ & 5.699722 & 6.614316 & 6.948549 \\
\hline $\mathrm{C}$ & 3.125115 & 8.074588 & 5.700433 \\
\hline $\mathrm{H}$ & 3.163276 & 7.015100 & 5.401628 \\
\hline $\mathrm{H}$ & 2.884915 & 8.083710 & 6.778602 \\
\hline $\mathrm{H}$ & 2.261765 & 8.524674 & 5.187488 \\
\hline $\mathrm{C}$ & 3.448643 & 11.195010 & 5.059479 \\
\hline $\mathrm{H}$ & 2.490126 & 10.741147 & 4.765566 \\
\hline $\mathrm{H}$ & 3.274570 & 11.681826 & 6.035310 \\
\hline $\mathrm{H}$ & 3.652078 & 12.008908 & 4.343867 \\
\hline $\mathrm{C}$ & 6.561955 & 11.830145 & 4.903221 \\
\hline $\mathrm{H}$ & 6.803932 & 12.269916 & 5.887165 \\
\hline
\end{tabular}

$\begin{array}{llll}\mathrm{H} & 7.503701 & 11.826058 & 4.331270 \\ \mathrm{H} & 5.884281 & 12.541213 & 4.406943 \\ \mathrm{C} & 4.557012 & 10.181515 & 5.110524 \\ \mathrm{C} & 5.951421 & 10.463719 & 5.025293 \\ \mathrm{C} & 4.407638 & 8.793524 & 5.393559 \\ \mathrm{C} & 5.707456 & 8.219252 & 5.486326 \\ \mathrm{C} & 6.663134 & 9.248802 & 5.249846\end{array}$

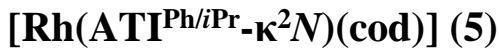

$\mathrm{TBE}=-7888.02$

$$
S^{0}=165.257
$$

$\begin{array}{llll}\mathrm{Rh} & 0.838721 & -0.451184 & -0.013381\end{array}$

N $\quad-0.861042 \quad-1.632677 \quad-0.028279$

$\begin{array}{llll}\text { C } & -2.041497 & -0.986698 & -0.009850\end{array}$

$\begin{array}{llll}\text { C } & -3.292164 & -1.661134 & -0.012971\end{array}$

$\begin{array}{llll}\text { C } & -4.610145 & -1.219648 & 0.006044\end{array}$

$\mathrm{H} \quad-3.212780 \quad-2.740826 \quad-0.032486$

$\begin{array}{llll}\mathrm{H} & -5.350482 & -2.023429 & -0.002913\end{array}$

$\begin{array}{llll}\mathrm{N} & -0.652839 & 0.937619 & -0.000732\end{array}$

$\begin{array}{llll}\text { C } & -1.924590 & 0.491919 & 0.006475\end{array}$

$\begin{array}{llll}\text { C } & -4.380808 & 1.246570 & 0.054012\end{array}$

$\begin{array}{llll}\text { C } & -3.000588 & 1.412548 & 0.037407\end{array}$

$\begin{array}{llll}\mathrm{H} & -4.950003 & 2.178691 & 0.081837\end{array}$

$\mathrm{H} \quad-2.676446 \quad 2.452464 \quad 0.052610$

$\begin{array}{llll}\text { C } & -0.774119 & -3.111136 & -0.064094\end{array}$

$\begin{array}{llll}\mathrm{H} & 0.299967 & -3.313179 & -0.073580\end{array}$

$\begin{array}{llll}\text { C } & -1.280677 & -3.730838 & -1.377923\end{array}$

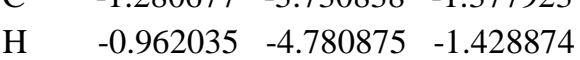

$\begin{array}{llll}\mathrm{H} & -0.962035 & -4.780875 & -1.428874 \\ \mathrm{H} & -2.369242 & -3.705027 & -1.495628\end{array}$

$\begin{array}{llll}\mathrm{H} & -2.369242 & -3.705027 & -1.495628 \\ \mathrm{H} & -0.838533 & -3.194180 & -2.227128\end{array}$

$\begin{array}{llll}\mathrm{H} & -0.838533 & -3.194180 & -2.227128 \\ \mathrm{H} & -1.275044 & -3.802335 & 1.216698\end{array}$

$\begin{array}{llll}\mathrm{C} & -1.275044 & -3.802335 & 1.216698 \\ \mathrm{H} & -2.364685 & -3.812448 & 1.326414\end{array}$

$\begin{array}{llll}\mathrm{H} & -2.364685 & -3.812448 & 1.326414 \\ \mathrm{H} & -0.928254 & -4.844600 & 1.223096\end{array}$

$\begin{array}{llll}\mathrm{H} & -0.928254 & -4.844600 & 1.223096 \\ \mathrm{C} & -0.856378 & -3.294126 & 2.094923\end{array}$

$\begin{array}{llll}\mathrm{H} & -0.856378 & -3.294126 & 2.094923 \\ \mathrm{C} & -5.130825 & 0.073735 & 0.038332\end{array}$

$\begin{array}{llll}\mathrm{C} & -5.130825 & 0.073735 & 0.038332 \\ \mathrm{H} & -6.216816 & 0.172895 & 0.053298\end{array}$

$\begin{array}{llll}\text { C } & 2.290716 & -1.936200 & -0.574333\end{array}$

$\begin{array}{llll}\text { C } & 3.496091 & -1.376098 & -1.320661\end{array}$

$\begin{array}{llll}\mathrm{H} & 4.385707 & -1.422268 & -0.678492\end{array}$

$\begin{array}{llll}\mathrm{H} & 3.715308 & -2.012282 & -2.188603\end{array}$

$\begin{array}{llll}\text { C } & 3.236127 & 0.070050 & -1.789773\end{array}$

$\begin{array}{llll}\mathrm{H} & 2.734882 & 0.044634 & -2.767784\end{array}$

$\begin{array}{lllll}\mathrm{H} & 4.186431 & 0.612933 & -1.941573\end{array}$

$\begin{array}{llll}\text { C } & 2.335078 & 0.828720 & -0.836909\end{array}$

$\begin{array}{llll}\text { C } & 2.456667 & 0.851124 & 0.568670\end{array}$

$\begin{array}{llll}\text { C } & 3.562993 & 0.147110 & 1.345156\end{array}$

$\begin{array}{llll}\mathrm{H} & 4.471274 & 0.089576 & 0.730291\end{array}$

$\begin{array}{llll}\mathrm{H} & 3.829701 & 0.749389 & 2.223921\end{array}$

$\begin{array}{llll}\text { C } & 3.122162 & -1.260384 & 1.799289\end{array}$

$\begin{array}{llll}\mathrm{H} & 2.615811 & -1.180453 & 2.771477\end{array}$

$\begin{array}{llll}\mathrm{H} & 3.997117 & -1.916397 & 1.957412\end{array}$

$\begin{array}{llll}\text { C } & 2.144416 & -1.898648 & 0.832200\end{array}$

H $\quad 1.752953 \quad-2.719784-1.115171$ 


$\begin{array}{lrrc}\mathrm{H} & 1.817835 & 1.675629 & -1.295911 \\ \mathrm{H} & 2.002308 & 1.701594 & 1.081525 \\ \mathrm{H} & 1.523003 & -2.671568 & 1.295489 \\ \mathrm{C} & -0.398620 & 2.335606 & 0.018915 \\ \mathrm{C} & -0.191305 & 2.995493 & 1.237452 \\ \mathrm{C} & -0.298526 & 3.054770 & -1.180038 \\ \mathrm{C} & 0.113597 & 4.356562 & 1.254609 \\ \mathrm{H} & -0.271011 & 2.421951 & 2.160506 \\ \mathrm{C} & 0.008854 & 4.415125 & -1.157916 \\ \mathrm{H} & -0.465882 & 2.528961 & -2.119733 \\ \mathrm{C} & 0.218027 & 5.070399 & 0.058163 \\ \mathrm{H} & 0.274084 & 4.860935 & 2.207500 \\ \mathrm{H} & 0.087188 & 4.965381 & -2.095564 \\ \mathrm{H} & 0.462466 & 6.131947 & 0.073161\end{array}$

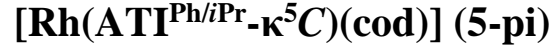

$\mathrm{TBE}=-7855.64$

$S^{0}=170.047$

$\begin{array}{lrcc}\mathrm{Rh} & 1.144429 & 0.772727 & 0.382700 \\ \mathrm{~N} & -3.262539 & 0.982761 & -0.288338 \\ \mathrm{C} & -2.037230 & 0.881813 & 0.075683 \\ \mathrm{C} & -1.067291 & 1.998350 & 0.127464 \\ \mathrm{C} & -0.273558 & 2.393802 & 1.201432 \\ \mathrm{H} & -1.201568 & 2.753888 & -0.646523 \\ \mathrm{H} & 0.083378 & 3.426455 & 1.192382 \\ \mathrm{~N} & -2.023083 & -1.490749 & -0.247148 \\ \mathrm{C} & -1.502796 & -0.490073 & 0.375019 \\ \mathrm{C} & 0.162559 & 0.186208 & 2.320641 \\ \mathrm{C} & -0.324425 & -0.627153 & 1.248513 \\ \mathrm{H} & 0.672082 & -0.313995 & 3.146019 \\ \mathrm{H} & -0.029561 & -1.673963 & 1.346672 \\ \mathrm{C} & -3.843172 & 2.274430 & -0.640274 \\ \mathrm{H} & -3.299679 & 3.119026 & -0.174247 \\ \mathrm{C} & -5.290389 & 2.303629 & -0.138118 \\ \mathrm{H} & -5.769908 & 3.257902 & -0.396324 \\ \mathrm{H} & -5.862227 & 1.483245 & -0.591660 \\ \mathrm{H} & -5.324854 & 2.179654 & 0.951756 \\ \mathrm{C} & -3.781811 & 2.433192 & -2.167818 \\ \mathrm{H} & -4.296261 & 1.591321 & -2.649616 \\ \mathrm{H} & -4.267574 & 3.368339 & -2.477195 \\ \mathrm{H} & -2.743534 & 2.442632 & -2.525313 \\ \mathrm{C} & 0.213700 & 1.593138 & 2.287267 \\ \mathrm{H} & 0.751073 & 2.104272 & 3.086765 \\ \mathrm{C} & 3.157036 & 1.610765 & 0.493746 \\ \mathrm{C} & 4.237575 & 0.593697 & 0.135589 \\ \mathrm{H} & 4.689438 & 0.877187 & -0.825082 \\ \mathrm{H} & 5.044954 & 0.649153 & 0.877543 \\ \mathrm{C} & 3.688993 & -0.845065 & 0.073468 \\ \mathrm{H} & 3.760595 & -1.297801 & 1.072073 \\ \mathrm{H} & 4.303039 & -1.475523 & -0.594968 \\ \mathrm{C} & 2.230549 & -0.901311 & -0.339192 \\ \mathrm{C} & 1.654567 & -0.208660 & -1.443540 \\ \mathrm{C} & 2.471403 & 0.648779 & -2.407704 \\ \mathrm{H} & 3.491119 & 0.244704 & -2.477758\end{array}$

$\begin{array}{cccc}\mathrm{H} & 2.044793 & 0.569392 & -3.416018 \\ \mathrm{C} & 2.502259 & 2.130077 & -1.979066 \\ \mathrm{H} & 1.637250 & 2.643767 & -2.420833 \\ \mathrm{H} & 3.399349 & 2.638916 & -2.375117 \\ \mathrm{C} & 2.408022 & 2.313422 & -0.476128 \\ \mathrm{H} & 3.259898 & 2.084106 & 1.473258 \\ \mathrm{H} & 1.724332 & -1.823699 & -0.039786 \\ \mathrm{H} & 0.735922 & -0.632067 & -1.856125 \\ \mathrm{H} & 2.011355 & 3.286505 & -0.174857 \\ \mathrm{C} & -1.546725 & -2.793568 & -0.078377 \\ \mathrm{C} & -2.088101 & -3.630538 & 0.915447 \\ \mathrm{C} & -0.570076 & -3.318240 & -0.945498 \\ \mathrm{C} & -1.646334 & -4.946318 & 1.046133 \\ \mathrm{H} & -2.856631 & -3.229498 & 1.575481 \\ \mathrm{C} & -0.131903 & -4.633835 & -0.802625 \\ \mathrm{H} & -0.163682 & -2.679663 & -1.728669 \\ \mathrm{C} & -0.663288 & -5.456709 & 0.193983 \\ \mathrm{H} & -2.074775 & -5.578586 & 1.824498 \\ \mathrm{H} & 0.631605 & -5.019083 & -1.479099 \\ \mathrm{H} & -0.321921 & -6.485704 & 0.299553\end{array}$

$\left[\mathrm{NaW}\left(\mathrm{ATI}^{\mathrm{Ph} / i \mathrm{Pr}}-2 \kappa^{2} N\right)(\mathrm{py}-2 \kappa N)\left(\mu_{2}-\mathrm{CO}-\right.\right.$ $\left.1 \kappa O, 2 \kappa C)_{2}(\mathrm{CO}-2 \kappa C)(p y-1 \kappa N)_{2}\right](9)$

$\mathrm{TBE}=-11405.70$

\begin{tabular}{lrrr}
\multicolumn{5}{l}{$S^{0}=252.144$} & & \\
$\mathrm{~W}$ & 0.664570 & 0.086219 & 0.484957 \\
$\mathrm{O}$ & -0.486568 & 3.010581 & 0.418756 \\
$\mathrm{C}$ & -0.741253 & -0.271334 & 1.774304 \\
$\mathrm{O}$ & -1.722861 & -0.501485 & 2.439773 \\
$\mathrm{C}$ & 2.712049 & -1.989601 & -0.626045 \\
$\mathrm{C}$ & 4.240003 & -0.544803 & -2.116860 \\
$\mathrm{H}$ & 4.449935 & 0.501037 & -2.343335 \\
$\mathrm{C}$ & 5.134355 & -1.454267 & -2.669613 \\
$\mathrm{H}$ & 5.945522 & -1.001207 & -3.245368 \\
$\mathrm{C}$ & 3.090737 & -0.720298 & -1.302351 \\
$\mathrm{C}$ & 3.168959 & -3.263765 & -1.078231 \\
$\mathrm{H}$ & 2.603420 & -4.107276 & -0.687247 \\
$\mathrm{C}$ & 4.196888 & -3.634102 & -1.937810 \\
$\mathrm{H}$ & 4.298690 & -4.713589 & -2.078201 \\
$\mathrm{C}$ & 5.123391 & -2.848760 & -2.627916 \\
$\mathrm{H}$ & 5.893337 & -3.371350 & -3.196806 \\
$\mathrm{~N}$ & 1.842535 & -1.835119 & 0.381359 \\
$\mathrm{~N}$ & 2.305493 & 0.316746 & -0.973896 \\
$\mathrm{Na}$ & -3.238042 & -0.393681 & 0.599128 \\
$\mathrm{O}$ & -1.719965 & -0.719546 & -1.376280 \\
$\mathrm{C}$ & -0.740511 & -0.419233 & -0.750736 \\
$\mathrm{~N}$ & 2.247922 & 0.669197 & 2.091485 \\
$\mathrm{C}$ & -0.047007 & 1.911245 & 0.434549 \\
$\mathrm{C}$ & 1.866292 & 1.332687 & 3.207030 \\
$\mathrm{H}$ & 0.805170 & 1.569770 & 3.263623 \\
$\mathrm{~N}$ & -4.440383 & 1.652611 & 0.164946 \\
$\mathrm{C}$ & 1.536194 & -2.993756 & 1.242020 \\
$\mathrm{H}$ & 2.330716 & -3.745591 & 1.119688
\end{tabular}




\begin{tabular}{|c|c|c|c|}
\hline C & 3.557527 & 0.353993 & 1.970335 \\
\hline H & 3.835699 & -0.179964 & 1.065490 \\
\hline C & 4.505920 & 0.681952 & 2.933890 \\
\hline H & 5.545914 & 0.400655 & 2.775964 \\
\hline 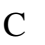 & 2.459018 & 1.564306 & -1.634059 \\
\hline $\mathrm{C}$ & 4.103809 & 1.361216 & 4.082110 \\
\hline H & 4.823364 & 1.630085 & 4.854766 \\
\hline C & 2.915412 & 2.682536 & -0.923505 \\
\hline $\mathrm{H}$ & 3.175086 & 2.563765 & 0.127397 \\
\hline C & 2.752421 & 1.689586 & 4.214732 \\
\hline $\mathrm{H}$ & 2.381504 & 2.220386 & 5.090097 \\
\hline C & 3.003430 & 3.924496 & -1.551401 \\
\hline $\mathrm{H}$ & 3.354690 & 4.788968 & -0.987563 \\
\hline C & 2.174511 & 2.952057 & -3.600505 \\
\hline $\mathrm{H}$ & 1.874422 & 3.054991 & -4.643654 \\
\hline C & 2.087647 & 1.707848 & -2.979122 \\
\hline $\mathrm{H}$ & 1.721403 & 0.833359 & -3.515764 \\
\hline C & 2.635052 & 4.065527 & -2.891391 \\
\hline $\mathrm{H}$ & 2.697788 & 5.038562 & -3.378231 \\
\hline C & 1.555054 & -2.594355 & 2.721623 \\
\hline $\mathrm{H}$ & 2.517599 & -2.138500 & 2.985360 \\
\hline $\mathrm{H}$ & 1.407953 & -3.485733 & 3.346839 \\
\hline $\mathrm{H}$ & 0.761640 & -1.872957 & 2.947758 \\
\hline $\mathrm{C}$ & 0.197502 & -3.625719 & 0.839998 \\
\hline $\mathrm{H}$ & -0.610934 & -2.899776 & 0.992651 \\
\hline $\mathrm{H}$ & -0.008491 & -4.518727 & 1.445969 \\
\hline $\mathrm{H}$ & 0.202007 & -3.908976 & -0.220450 \\
\hline $\mathrm{C}$ & -3.781542 & 2.828020 & 0.166347 \\
\hline $\mathrm{H}$ & -2.722545 & 2.800524 & 0.427299 \\
\hline C & -5.741372 & 1.666611 & -0.178653 \\
\hline $\mathrm{H}$ & -6.246205 & 0.698126 & -0.185961 \\
\hline C & -4.391866 & 4.038601 & -0.162904 \\
\hline $\mathrm{H}$ & -3.806004 & 4.956212 & -0.149104 \\
\hline $\mathrm{C}$ & -5.741624 & 4.041063 & -0.508368 \\
\hline $\mathrm{H}$ & -6.247792 & 4.970263 & -0.769721 \\
\hline $\mathrm{C}$ & -6.431497 & 2.828072 & -0.519255 \\
\hline $\mathrm{H}$ & -7.485649 & 2.778494 & -0.788392 \\
\hline $\mathrm{N}$ & -4.894757 & -1.912175 & -0.347112 \\
\hline $\mathrm{C}$ & -6.083040 & -2.279215 & 0.166199 \\
\hline $\mathrm{H}$ & -6.301105 & -1.927624 & 1.176996 \\
\hline C & -7.006406 & -3.063791 & -0.522458 \\
\hline $\mathrm{H}$ & -7.952782 & -3.330995 & -0.054300 \\
\hline $\mathrm{C}$ & -6.686216 & -3.493999 & -1.810421 \\
\hline $\mathrm{H}$ & -7.382471 & -4.109544 & -2.379567 \\
\hline C & -5.457697 & -3.121907 & -2.353304 \\
\hline $\mathrm{H}$ & -5.163627 & -3.434655 & -3.354087 \\
\hline C & -4.594855 & -2.333880 & -1.591426 \\
\hline $\mathrm{H}$ & -3.623517 & -2.022915 & -1.979478 \\
\hline
\end{tabular}

$\left[\mathrm{NaW}\left(\mathrm{ATI}^{\mathrm{Ph} / \mathrm{Pr}}-1 \kappa^{2} N, 2 \kappa^{5} C\right)(\mathrm{CO}-\right.$ $\left.\left.\mathbf{2 \kappa}_{3}\right)_{3}(\mathrm{py}-1 \kappa N)_{3}\right](10)$

$\mathrm{TBE}=-11383.97$ $S^{0}=265.740$

\begin{tabular}{|c|c|c|c|}
\hline & -3.244559 & 0.374082 & \\
\hline $\mathrm{O}$ & -5.124688 & 1.736134 & 2.049 \\
\hline C & -4.522032 & 1.034337 & -1.398689 \\
\hline $\mathrm{O}$ & -5.295812 & 1.407068 & -2.196791 \\
\hline $\mathrm{C}$ & -0.675340 & -1.329218 & -0.854324 \\
\hline C & -1.708155 & -0.780678 & 1.433802 \\
\hline $\mathrm{H}$ & -1.485532 & -0.364421 & 2.416061 \\
\hline $\mathrm{C}$ & -2.964911 & -1.446636 & 1.367393 \\
\hline $\mathrm{H}$ & -3.500130 & -1.523964 & 2.314866 \\
\hline $\mathrm{C}$ & -0.560623 & -0.807063 & 0.554610 \\
\hline $\mathrm{C}$ & -1.970853 & -1.193105 & -1.513461 \\
\hline $\mathrm{H}$ & -1.894577 & -1.040405 & -2.590254 \\
\hline $\mathrm{C}$ & -3.235629 & -1.698320 & -1.101512 \\
\hline $\mathrm{H}$ & -3.957593 & -1.912631 & -1.890872 \\
\hline $\mathrm{C}$ & -3.657172 & -1.942724 & 0.229128 \\
\hline $\mathrm{H}$ & -4.642747 & -2.377007 & 0.386080 \\
\hline $\mathrm{N}$ & 0.427641 & -1.690760 & -1.438085 \\
\hline $\mathrm{N}$ & 0.608260 & -0.326976 & 0.890529 \\
\hline $\mathrm{Na}$ & 2.412925 & -0.209449 & -0.709645 \\
\hline $\mathrm{O}$ & -1.598806 & 3.039780 & -0.215365 \\
\hline C & -2.246990 & 2.060145 & -0.173700 \\
\hline $\mathrm{C}$ & -4.408433 & 1.243009 & 1.264866 \\
\hline $\mathrm{N}$ & 4.262599 & 1.179769 & 0.300560 \\
\hline $\mathrm{C}$ & 0.404327 & -2.237304 & -2.801806 \\
\hline $\mathrm{H}$ & -0.095828 & -1.528348 & -3.488615 \\
\hline $\mathrm{N}$ & 3.454412 & -2.081568 & 0.583509 \\
\hline $\mathrm{C}$ & 0.851025 & 0.285972 & 2.129222 \\
\hline $\mathrm{C}$ & 0.467687 & 1.620594 & 2.362290 \\
\hline $\mathrm{H}$ & -0.128396 & 2.137994 & 1.612457 \\
\hline $\mathrm{C}$ & 2.530620 & -3.046992 & 0.752006 \\
\hline $\mathrm{H}$ & 1.710617 & -3.037923 & 0.029112 \\
\hline $\mathrm{C}$ & 4.455814 & -2.015685 & 1.476858 \\
\hline $\mathrm{H}$ & 5.184514 & -1.217497 & 1.325998 \\
\hline C & 3.621425 & -3.890414 & 2.718116 \\
\hline $\mathrm{H}$ & 3.682717 & -4.589458 & 3.552059 \\
\hline $\mathrm{C}$ & 0.867508 & 2.273214 & 3.528353 \\
\hline $\mathrm{H}$ & 0.566447 & 3.308418 & 3.689673 \\
\hline C & 2.016333 & 0.282772 & 4.262927 \\
\hline $\mathrm{H}$ & 2.618932 & -0.246640 & 5.001813 \\
\hline $\mathrm{C}$ & 1.622380 & -0.378549 & 3.100322 \\
\hline $\mathrm{H}$ & 1.919074 & -1.410563 & 2.921412 \\
\hline $\mathrm{C}$ & 2.577500 & -3.970084 & 1.796510 \\
\hline $\mathrm{H}$ & 1.798999 & -4.726171 & 1.888307 \\
\hline C & 1.649874 & 1.613586 & 4.481708 \\
\hline $\mathrm{H}$ & 1.961806 & 2.129035 & 5.389535 \\
\hline $\mathrm{C}$ & 1.850249 & -2.417394 & -3.275315 \\
\hline $\mathrm{H}$ & 2.380286 & -1.458250 & -3.309038 \\
\hline $\mathrm{H}$ & 1.882179 & -2.852434 & -4.282954 \\
\hline $\mathrm{H}$ & 2.389674 & -3.087686 & -2.590783 \\
\hline $\mathrm{C}$ & -0.332363 & -3.586568 & -2.850885 \\
\hline $\mathrm{H}$ & 0.169074 & -4.303642 & -2.185695 \\
\hline $\mathrm{H}$ & -0.319588 & -3.995888 & -3.869776 \\
\hline $\mathrm{H}$ & -1.374515 & -3.493123 & -2.527064 \\
\hline & 4.064228 & 1.618968 & 1.557047 \\
\hline
\end{tabular}




$\begin{array}{lrrr}\mathrm{H} & 3.028496 & 1.732838 & 1.876252 \\ \mathrm{C} & 5.535073 & 1.044922 & -0.114200 \\ \mathrm{H} & 5.674423 & 0.689718 & -1.137600 \\ \mathrm{C} & 4.580468 & -2.891056 & 2.555606 \\ \mathrm{H} & 5.410063 & -2.783503 & 3.252954 \\ \mathrm{C} & 5.106462 & 1.913377 & 2.437156 \\ \mathrm{H} & 4.874981 & 2.251692 & 3.445775 \\ \mathrm{C} & 6.419864 & 1.757460 & 1.998606 \\ \mathrm{H} & 7.258797 & 1.975364 & 2.659341 \\ \mathrm{C} & 6.639716 & 1.319468 & 0.691196 \\ \mathrm{H} & 7.647641 & 1.188617 & 0.299649 \\ \mathrm{~N} & 1.506457 & 1.085653 & -2.705410 \\ \mathrm{C} & 1.791091 & 1.081411 & -4.020331 \\ \mathrm{H} & 2.824504 & 0.851691 & -4.289393 \\ \mathrm{C} & 0.845312 & 1.336389 & -5.011483 \\ \mathrm{H} & 1.134506 & 1.309926 & -6.061341 \\ \mathrm{C} & -0.467575 & 1.616801 & -4.627087 \\ \mathrm{H} & -1.235950 & 1.814415 & -5.374206 \\ \mathrm{C} & -0.775141 & 1.642277 & -3.268457 \\ \mathrm{H} & -1.781056 & 1.845133 & -2.905111 \\ \mathrm{C} & 0.241007 & 1.375474 & -2.351845 \\ \mathrm{H} & 0.022705 & 1.373817 & -1.284407\end{array}$

$\left[\mathrm{NaW}\left(\mathrm{ATI}^{\mathrm{Pr} / \mathrm{Pr}}-1 \kappa^{5} \mathrm{C}, 2 \mathrm{\kappa}^{2} N\right)(\mathrm{CO}-\right.$ $2 \kappa C) 3(p y-1 \kappa N) 2(p y-2 \kappa N)](11)$

$\mathrm{TBE}=-11398.20$

\begin{tabular}{|c|c|c|c|}
\hline \multicolumn{4}{|c|}{$S^{0}=265.360$} \\
\hline W & -1.966509 & -0.829787 & 0.165855 \\
\hline $\mathrm{O}$ & -4.340388 & 0.701646 & 1.544130 \\
\hline $\mathrm{C}$ & -2.564195 & -2.430255 & 1.142903 \\
\hline $\mathrm{O}$ & -2.902916 & -3.390731 & 1.741186 \\
\hline $\mathrm{C}$ & 0.333316 & -0.736489 & -1.923449 \\
\hline $\mathrm{C}$ & 0.414208 & 1.778917 & -2.568479 \\
\hline $\mathrm{H}$ & -0.022215 & 2.738680 & -2.291090 \\
\hline $\mathrm{C}$ & 1.395136 & 1.850426 & -3.553152 \\
\hline $\mathrm{H}$ & 1.617867 & 2.863335 & -3.898751 \\
\hline C & -0.142720 & 0.673481 & -1.846939 \\
\hline $\mathrm{C}$ & 1.187784 & -1.216707 & -2.979026 \\
\hline $\mathrm{H}$ & 1.229863 & -2.300024 & -3.060911 \\
\hline $\mathrm{C}$ & 1.949176 & -0.555171 & -3.938036 \\
\hline $\mathrm{H}$ & 2.503236 & -1.217709 & -4.608860 \\
\hline $\mathrm{C}$ & 2.116691 & 0.820432 & -4.184103 \\
\hline $\mathrm{H}$ & 2.792434 & 1.105524 & -4.990813 \\
\hline $\mathrm{N}$ & -0.172495 & -1.529897 & -0.973427 \\
\hline $\mathrm{N}$ & -1.121565 & 0.844952 & -0.952904 \\
\hline $\mathrm{Na}$ & 3.100079 & 0.750180 & -1.629633 \\
\hline $\mathrm{O}$ & -3.885774 & -1.660379 & -2.195412 \\
\hline $\mathrm{C}$ & -3.174014 & -1.343612 & -1.318709 \\
\hline $\mathrm{N}$ & -0.588340 & -0.270015 & 1.856854 \\
\hline $\mathrm{C}$ & -3.468649 & 0.105395 & 1.024037 \\
\hline $\mathrm{C}$ & -1.013823 & -0.345224 & 3.148881 \\
\hline $\mathrm{H}$ & -2.024915 & -0.725225 & 3.279749 \\
\hline $\mathrm{N}$ & 3.089923 & 2.424762 & 0.117312 \\
\hline
\end{tabular}

\begin{tabular}{|c|c|c|c|}
\hline & 0.339442 & -2.910764 & -0.844315 \\
\hline & 1.297739 & -2.986209 & -1.378179 \\
\hline C & 0.671589 & 0.199337 & 1.658476 \\
\hline $\mathrm{H}$ & 0.995600 & 0.236713 & 0.621217 \\
\hline $\mathrm{C}$ & 1.499612 & 0.617378 & 2.692322 \\
\hline $\mathrm{H}$ & 2.484694 & 1.016052 & 2.459521 \\
\hline $\mathrm{C}$ & -1.747121 & 2.110629 & -0.789807 \\
\hline $\mathrm{C}$ & 1.051418 & 0.543453 & 4.009934 \\
\hline H & 1.679208 & 0.867692 & 4.838648 \\
\hline C & -1.587470 & 2.807334 & 0.416230 \\
\hline $\mathrm{H}$ & -0.978103 & 2.360705 & 1.199941 \\
\hline C & -0.236672 & 0.039799 & 4.228849 \\
\hline $\mathrm{H}$ & -0.647064 & -0.048554 & 5.233471 \\
\hline C & -2.245931 & 4.019218 & 0.617724 \\
\hline $\mathrm{H}$ & -2.133085 & 4.542641 & 1.567816 \\
\hline C & -3.245584 & 3.843702 & -1.572557 \\
\hline $\mathrm{H}$ & -3.910263 & 4.233807 & -2.343297 \\
\hline $\mathrm{C}$ & -2.586472 & 2.633494 & -1.783129 \\
\hline $\mathrm{H}$ & -2.729243 & 2.065140 & -2.701158 \\
\hline C & -3.074063 & 4.545960 & -0.376785 \\
\hline $\mathrm{H}$ & -3.601484 & 5.484809 & -0.211523 \\
\hline $\mathrm{C}$ & 0.651465 & -3.229843 & 0.619612 \\
\hline $\mathrm{H}$ & 1.334519 & -2.480637 & 1.039150 \\
\hline $\mathrm{H}$ & 1.136163 & -4.213603 & 0.682832 \\
\hline $\mathrm{H}$ & -0.257575 & -3.247752 & 1.228815 \\
\hline $\mathrm{C}$ & -0.640881 & -3.911411 & -1.464714 \\
\hline $\mathrm{H}$ & -1.593507 & -3.888204 & -0.923270 \\
\hline $\mathrm{H}$ & -0.229309 & -4.928860 & -1.414820 \\
\hline $\mathrm{H}$ & -0.842657 & -3.660475 & -2.513919 \\
\hline $\mathrm{C}$ & 1.916276 & 3.042738 & 0.358144 \\
\hline $\mathrm{H}$ & 1.104096 & 2.830952 & -0.337278 \\
\hline $\mathrm{C}$ & 4.101964 & 2.662160 & 0.975969 \\
\hline $\mathrm{H}$ & 5.045200 & 2.153764 & 0.763884 \\
\hline C & 1.710638 & 3.898423 & 1.439034 \\
\hline $\mathrm{H}$ & 0.731872 & 4.354651 & 1.575920 \\
\hline $\mathrm{C}$ & 2.762825 & 4.130595 & 2.320173 \\
\hline $\mathrm{H}$ & 2.635653 & 4.788674 & 3.179201 \\
\hline $\mathrm{C}$ & 3.986164 & 3.498069 & 2.083761 \\
\hline $\mathrm{H}$ & 4.838742 & 3.648854 & 2.744133 \\
\hline $\mathrm{N}$ & 3.859530 & -1.190840 & -0.432594 \\
\hline $\mathrm{C}$ & 3.872666 & -2.391781 & -1.042790 \\
\hline $\mathrm{H}$ & 3.715697 & -2.381960 & -2.122287 \\
\hline $\mathrm{C}$ & 4.050047 & -3.592530 & -0.358669 \\
\hline $\mathrm{H}$ & 4.041186 & -4.534422 & -0.904483 \\
\hline $\mathrm{C}$ & 4.216878 & -3.558516 & 1.024292 \\
\hline $\mathrm{H}$ & 4.342816 & -4.480042 & 1.591543 \\
\hline $\mathrm{C}$ & 4.204854 & -2.322451 & 1.669009 \\
\hline $\mathrm{H}$ & 4.316133 & -2.244606 & 2.748950 \\
\hline $\mathrm{C}$ & 4.026544 & -1.173810 & 0.902635 \\
\hline $\mathrm{H}$ & 4.003176 & -0.192370 & 1.376298 \\
\hline
\end{tabular}


[NaW(ATI $\left.{ }^{\mathrm{Ph} / \mathrm{Pr}_{-}}-1 \mathrm{\kappa}^{5} \mathrm{C}, \mathbf{2} \kappa^{2} N\right)(\mathrm{CO}-$ $2 \kappa C)_{2}\left(\mu_{2} \mathrm{CO}-1 \kappa C O, 2 \kappa C\right)(\mathrm{py}-1 \kappa N)_{2}(\mathrm{py}-$ $2 \kappa N)](12)$

\begin{tabular}{|c|c|c|c|}
\hline \multicolumn{4}{|c|}{$\mathrm{TBE}=-11409.8$} \\
\hline $\mathrm{W}$ & -0.958908 & -0.718897 & -0.849969 \\
\hline $\mathrm{O}$ & -2.109195 & 0.770412 & -3.365151 \\
\hline $\mathrm{C}$ & -1.248651 & -2.386988 & -1.846770 \\
\hline $\mathrm{O}$ & -1.413955 & -3.398400 & -2.431547 \\
\hline $\mathrm{C}$ & 0.526947 & -0.558730 & 1.886912 \\
\hline $\mathrm{C}$ & 0.511030 & 1.976292 & 2.418791 \\
\hline $\mathrm{H}$ & 0.016278 & 2.900852 & 2.123762 \\
\hline $\mathrm{C}$ & 1.428861 & 2.117355 & 3.452757 \\
\hline $\mathrm{H}$ & 1.518516 & 3.135842 & 3.840361 \\
\hline $\mathrm{C}$ & 0.120371 & 0.856653 & 1.628890 \\
\hline C & 1.563349 & -0.923689 & 2.804874 \\
\hline $\mathrm{H}$ & 1.835456 & -1.976154 & 2.771261 \\
\hline $\mathrm{C}$ & 2.312568 & -0.188077 & 3.720124 \\
\hline $\mathrm{H}$ & 3.047404 & -0.779509 & 4.273908 \\
\hline $\mathrm{C}$ & 2.267909 & 1.169037 & 4.045908 \\
\hline $\mathrm{H}$ & 2.942207 & 1.517503 & 4.828616 \\
\hline $\mathrm{N}$ & -0.121195 & -1.455700 & 1.132035 \\
\hline $\mathrm{N}$ & -0.670175 & 0.998108 & 0.553904 \\
\hline $\mathrm{Na}$ & 2.708814 & 0.355209 & -0.103563 \\
\hline $\mathrm{O}$ & 1.856175 & -0.545800 & -2.213021 \\
\hline $\mathrm{C}$ & 0.763965 & -0.623417 & -1.702443 \\
\hline $\mathrm{N}$ & -3.043484 & -0.803615 & 0.156079 \\
\hline $\mathrm{C}$ & -1.684014 & 0.197859 & -2.425739 \\
\hline $\mathrm{C}$ & -4.151807 & -1.014110 & -0.592310 \\
\hline $\mathrm{H}$ & -3.974693 & -1.156372 & -1.656966 \\
\hline $\mathrm{N}$ & 4.140624 & -1.547405 & 0.193440 \\
\hline $\mathrm{C}$ & -0.008227 & -2.890755 & 1.466569 \\
\hline $\mathrm{H}$ & 0.273961 & -2.983353 & 2.527999 \\
\hline $\mathrm{C}$ & -3.215474 & -0.618964 & 1.484365 \\
\hline $\mathrm{H}$ & -2.302747 & -0.463275 & 2.055694 \\
\hline $\mathrm{C}$ & -4.463322 & -0.634361 & 2.096813 \\
\hline $\mathrm{H}$ & -4.533822 & -0.478457 & 3.172546 \\
\hline $\mathrm{C}$ & -1.366559 & 2.210712 & 0.311563 \\
\hline C & -5.599551 & -0.851570 & 1.319193 \\
\hline $\mathrm{H}$ & -6.591524 & -0.869289 & 1.769186 \\
\hline $\mathrm{C}$ & -2.329214 & 2.681453 & 1.221396 \\
\hline
\end{tabular}

$\begin{array}{lrrr}\mathrm{H} & -2.488880 & 2.137367 & 2.151207 \\ \mathrm{C} & -5.431323 & -1.046072 & -0.053411 \\ \mathrm{H} & -6.282496 & -1.218886 & -0.709766 \\ \mathrm{C} & -3.076309 & 3.821830 & 0.930085 \\ \mathrm{H} & -3.828105 & 4.167309 & 1.640117 \\ \mathrm{C} & -1.918838 & 4.044837 & -1.174102 \\ \mathrm{H} & -1.758467 & 4.563303 & -2.119334 \\ \mathrm{C} & -1.177482 & 2.901486 & -0.891256 \\ \mathrm{H} & -0.466671 & 2.501897 & -1.608939 \\ \mathrm{C} & -2.872160 & 4.514063 & -0.266482 \\ \mathrm{H} & -3.461026 & 5.401954 & -0.495072 \\ \mathrm{C} & -1.368017 & -3.580853 & 1.322395 \\ \mathrm{H} & -2.122722 & -3.089523 & 1.948433 \\ \mathrm{H} & -1.283317 & -4.628930 & 1.639981 \\ \mathrm{H} & -1.716170 & -3.559716 & 0.284135 \\ \mathrm{C} & 1.064424 & -3.572533 & 0.609836 \\ \mathrm{H} & 0.823817 & -3.445899 & -0.452656 \\ \mathrm{H} & 1.117486 & -4.645484 & 0.837700 \\ \mathrm{H} & 2.050475 & -3.130118 & 0.792492 \\ \mathrm{C} & 4.143606 & -2.421812 & -0.832007 \\ \mathrm{H} & 3.705348 & -2.065535 & -1.765298 \\ \mathrm{C} & 4.632013 & -1.965981 & 1.374599 \\ \mathrm{H} & 4.601537 & -1.242532 & 2.190917 \\ \mathrm{C} & 4.627471 & -3.724412 & -0.716277 \\ \mathrm{H} & 4.592972 & -4.393409 & -1.574651 \\ \mathrm{C} & 5.125442 & -4.149031 & 0.513842 \\ \mathrm{H} & 5.497561 & -5.165123 & 0.642346 \\ \mathrm{C} & 5.130065 & -3.250637 & 1.581331 \\ \mathrm{H} & 5.505279 & -3.538783 & 2.562259 \\ \mathrm{~N} & 2.345183 & 2.599251 & -0.844932 \\ \mathrm{C} & 2.095214 & 2.735437 & -2.162523 \\ \mathrm{H} & 2.202905 & 1.832947 & -2.766282 \\ \mathrm{C} & 1.684792 & 3.937319 & -2.737000 \\ \mathrm{H} & 1.485773 & 3.985134 & -3.806292 \\ \mathrm{C} & 1.513837 & 5.050298 & -1.915636 \\ \mathrm{H} & 1.181262 & 6.001468 & -2.329859 \\ \mathrm{C} & 1.758762 & 4.917586 & -0.550335 \\ \mathrm{H} & 1.620696 & 5.753378 & 0.133382 \\ \mathrm{C} & 2.172443 & 3.680177 & -0.062426 \\ \mathrm{H} & 2.356292 & 3.535417 & 1.003140\end{array}$


Free energies $\left(\mathrm{G}^{\circ}\right.$, hartree), enthalpies $\left(\mathrm{H}^{\circ}\right.$, hartree), and cartesian coordinates $(\AA)$ of optimized structures. Differing from the other calculations presented in this work, these calculations were performed with the B3LYP functional and the 6-31G(d,p) $(H, C, N)$ and LanL2DZ (Rh) basis sets.

\begin{tabular}{|c|c|c|c|}
\hline \multicolumn{4}{|c|}{$\left[\mathrm{Rh}\left(\mathrm{ATI}^{t \mathrm{Bu} / t \mathrm{Bu}}-\kappa^{2} N\right)(\operatorname{cod})\right](13-\mathrm{sp})$} \\
\hline \multicolumn{4}{|c|}{$\mathrm{H}^{\circ}=-1115.960987$} \\
\hline \multicolumn{4}{|c|}{$G^{\circ}=-1116.043500$} \\
\hline $\mathrm{Rh}$ & -0.965370 & 0.000029 & 0.000046 \\
\hline $\mathrm{N}$ & 0.744415 & 1.420759 & -0.113915 \\
\hline $\mathrm{C}$ & 1.837917 & 0.721948 & 0.177002 \\
\hline $\mathrm{C}$ & 2.956791 & 1.170172 & 0.950506 \\
\hline $\mathrm{C}$ & 4.304545 & 0.831971 & 0.908209 \\
\hline $\mathrm{H}$ & 2.700695 & 1.928406 & 1.681634 \\
\hline $\mathrm{H}$ & 4.931622 & 1.352093 & 1.630789 \\
\hline $\mathrm{N}$ & 0.744399 & -1.420831 & 0.113875 \\
\hline $\mathrm{C}$ & 1.837932 & -0.722015 & -0.176966 \\
\hline $\mathrm{C}$ & 4.304615 & -0.831839 & -0.908013 \\
\hline $\mathrm{C}$ & 2.956865 & -1.170135 & -0.950401 \\
\hline $\mathrm{H}$ & 4.931741 & -1.351936 & -1.630569 \\
\hline $\mathrm{H}$ & 2.700947 & -1.928404 & -1.681544 \\
\hline $\mathrm{C}$ & 2.193766 & 3.410200 & -0.756918 \\
\hline $\mathrm{H}$ & 2.138148 & 4.490105 & -0.925555 \\
\hline $\mathrm{H}$ & 3.055491 & 3.219608 & -0.122011 \\
\hline $\mathrm{H}$ & 2.374760 & 2.925945 & -1.721816 \\
\hline $\mathrm{C}$ & 0.617251 & 3.581116 & 1.222290 \\
\hline $\mathrm{H}$ & 1.442932 & 3.394140 & 1.912366 \\
\hline $\mathrm{H}$ & 0.523521 & 4.667694 & 1.114227 \\
\hline $\mathrm{H}$ & -0.294599 & 3.208147 & 1.694875 \\
\hline $\mathrm{C}$ & 4.959295 & 0.000081 & 0.000095 \\
\hline $\mathrm{H}$ & 6.045754 & 0.000118 & 0.000102 \\
\hline $\mathrm{C}$ & -2.569254 & 1.226412 & -0.865382 \\
\hline $\mathrm{C}$ & -3.717745 & 0.427865 & -1.469561 \\
\hline $\mathrm{H}$ & -4.622167 & 0.556331 & -0.867159 \\
\hline $\mathrm{H}$ & -3.954546 & 0.838599 & -2.457008 \\
\hline $\mathrm{C}$ & -3.358096 & -1.062748 & -1.604750 \\
\hline $\mathrm{H}$ & -2.838473 & -1.221146 & -2.556320 \\
\hline $\mathrm{H}$ & -4.266196 & -1.684455 & -1.644701 \\
\hline C & -2.436835 & -1.531390 & -0.498293 \\
\hline $\mathrm{C}$ & -2.568931 & -1.226292 & 0.865422 \\
\hline $\mathrm{C}$ & -3.717515 & -0.427945 & 1.469710 \\
\hline $\mathrm{H}$ & -4.621999 & -0.556630 & 0.867440 \\
\hline $\mathrm{H}$ & -3.954116 & -0.838644 & 2.457220 \\
\hline $\mathrm{C}$ & -3.358172 & 1.062749 & 1.604805 \\
\hline $\mathrm{H}$ & -2.838443 & 1.221268 & 2.556295 \\
\hline $\mathrm{H}$ & -4.266407 & 1.684253 & 1.644905 \\
\hline $\mathrm{C}$ & -2.437158 & 1.531634 & 0.498245 \\
\hline $\mathrm{H}$ & -2.057435 & 1.864820 & -1.573133 \\
\hline $\mathrm{H}$ & -1.865683 & -2.414876 & -0.757991 \\
\hline $\mathrm{H}$ & -2.057196 & -1.864871 & 1.573067 \\
\hline $\mathrm{H}$ & -1.865865 & 2.415019 & 0.758004 \\
\hline $\mathrm{C}$ & 0.843068 & -2.929900 & 0.160687 \\
\hline
\end{tabular}

$\begin{array}{lrrr}\mathrm{C} & 0.617138 & -3.581108 & -1.222502 \\ \mathrm{H} & -0.294908 & -3.208390 & -1.694909 \\ \mathrm{H} & 1.442621 & -3.393832 & -1.912731 \\ \mathrm{H} & 0.523746 & -4.667718 & -1.114489 \\ \mathrm{C} & 2.193702 & -3.410296 & 0.756738 \\ \mathrm{H} & 3.055444 & -3.219809 & 0.121821 \\ \mathrm{H} & 2.374711 & -2.925941 & 1.721583 \\ \mathrm{H} & 2.138049 & -4.490180 & 0.925492 \\ \mathrm{C} & -0.214205 & -3.475648 & 1.144232 \\ \mathrm{H} & -0.009347 & -4.531571 & 1.344824 \\ \mathrm{H} & -0.164347 & -2.936065 & 2.094436 \\ \mathrm{H} & -1.229122 & -3.422530 & 0.757918 \\ \mathrm{C} & 0.843145 & 2.929851 & -0.160887 \\ \mathrm{C} & -0.214199 & 3.475395 & -1.144439 \\ \mathrm{H} & -0.164458 & 2.935541 & -2.094499 \\ \mathrm{H} & -1.229061 & 3.422355 & -0.757976 \\ \mathrm{H} & -0.009364 & 4.531264 & -1.345342\end{array}$

$\left[\operatorname{Rh}\left(\mathrm{ATI}^{t \mathrm{Bu} / \mathrm{tBu}}-\kappa^{5} \mathrm{C}\right)(\mathrm{cod})\right](13-\mathrm{pi})$

$\begin{array}{lrrr}\mathrm{H}^{\circ}=-1115.951822 & \\ \mathrm{G}^{\circ}=-1116.038514 & \\ \mathrm{Rh} & 1.535936 & -0.402416 & -0.317227 \\ \mathrm{~N} & -3.367830 & -0.493030 & 0.042030 \\ \mathrm{C} & -2.091954 & -0.556749 & -0.033864 \\ \mathrm{C} & -1.260908 & -1.763208 & 0.160191 \\ \mathrm{C} & -0.275831 & -2.227187 & -0.656942 \\ \mathrm{H} & -1.547446 & -2.403712 & 0.987840 \\ \mathrm{H} & 0.119726 & -3.216385 & -0.433508 \\ \mathrm{~N} & -1.960340 & 1.782177 & 0.252729 \\ \mathrm{C} & -1.391829 & 0.780781 & -0.301951 \\ \mathrm{C} & 0.210567 & -0.210767 & -2.128097 \\ \mathrm{C} & -0.200074 & 0.790964 & -1.199084 \\ \mathrm{H} & 0.681219 & 0.118016 & -3.051634 \\ \mathrm{H} & 0.060548 & 1.792545 & -1.520453 \\ \mathrm{C} & -4.295706 & -1.605244 & 0.320887 \\ \mathrm{C} & -4.082810 & -2.845798 & -0.572466 \\ \mathrm{H} & -4.910907 & -3.549463 & -0.433864 \\ \mathrm{H} & -4.063556 & -2.553378 & -1.627543 \\ \mathrm{H} & -3.153073 & -3.373726 & -0.352898 \\ \mathrm{C} & 0.331847 & -1.578385 & -1.802593 \\ \mathrm{H} & 0.843555 & -2.222122 & -2.512970 \\ \mathrm{C} & 3.547738 & -1.318000 & -0.593585 \\ \mathrm{C} & 4.654492 & -0.278294 & -0.464543 \\ \mathrm{H} & 5.235816 & -0.478195 & 0.441458 \\ \mathrm{H} & 5.353717 & -0.396104 & -1.299396 \\ \mathrm{C} & 4.123982 & 1.173087 & -0.453876 \\ \mathrm{H} & 4.043657 & 1.528386 & -1.487197\end{array}$




\begin{tabular}{lrrr}
$\mathrm{H}$ & 4.849530 & 1.838668 & 0.040213 \\
$\mathrm{C}$ & 2.754406 & 1.321175 & 0.182865 \\
$\mathrm{C}$ & 2.336409 & 0.736571 & 1.390575 \\
$\mathrm{C}$ & 3.237354 & -0.110910 & 2.283624 \\
$\mathrm{H}$ & 4.271890 & 0.238543 & 2.200910 \\
$\mathrm{H}$ & 2.954044 & 0.046118 & 3.329901 \\
$\mathrm{C}$ & 3.153099 & -1.620818 & 1.963812 \\
$\mathrm{H}$ & 2.316974 & -2.052574 & 2.525036 \\
$\mathrm{H}$ & 4.057313 & -2.138896 & 2.320544 \\
$\mathrm{C}$ & 2.908851 & -1.920977 & 0.496198 \\
$\mathrm{H}$ & 3.503183 & -1.842965 & -1.545399 \\
$\mathrm{H}$ & 2.208506 & 2.198869 & -0.151558 \\
$\mathrm{H}$ & 1.472669 & 1.183366 & 1.878512 \\
$\mathrm{H}$ & 2.432415 & -2.882672 & 0.310802 \\
$\mathrm{C}$ & -1.674553 & 3.217713 & 0.123918 \\
$\mathrm{C}$ & -2.040164 & 3.693807 & -1.299853 \\
$\mathrm{H}$ & -1.376005 & 3.273446 & -2.060334 \\
$\mathrm{H}$ & -3.063628 & 3.393430 & -1.542330 \\
$\mathrm{H}$ & -1.977138 & 4.785653 & -1.363495 \\
$\mathrm{C}$ & -0.232696 & 3.628696 & 0.489558 \\
$\mathrm{H}$ & 0.061786 & 3.183779 & 1.445349 \\
$\mathrm{H}$ & 0.495791 & 3.332955 & -0.267175 \\
$\mathrm{H}$ & -0.171315 & 4.717478 & 0.593198 \\
$\mathrm{C}$ & -2.631370 & 3.900026 & 1.126443 \\
$\mathrm{H}$ & -2.400021 & 3.581419 & 2.147776 \\
$\mathrm{H}$ & -2.550454 & 4.991738 & 1.075330 \\
$\mathrm{H}$ & -3.663616 & 3.609302 & 0.914170 \\
$\mathrm{C}$ & -5.696811 & -1.030483 & 0.020676 \\
$\mathrm{H}$ & -5.768817 & -0.738932 & -1.031732 \\
$\mathrm{H}$ & -6.481917 & -1.764812 & 0.233276 \\
$\mathrm{H}$ & -5.873822 & -0.136901 & 0.625530 \\
$\mathrm{C}$ & -4.233598 & -1.973397 & 1.819082 \\
$\mathrm{H}$ & -3.289526 & -2.450780 & 2.096393 \\
$\mathrm{H}$ & -4.351347 & -1.074606 & 2.431535 \\
$\mathrm{H}$ & -5.042348 & -2.668385 & 2.069766 \\
& & & \\
\hline
\end{tabular}

$\left[\operatorname{Rh}\left(\mathrm{ATI}^{t \mathrm{Bu} / \mathrm{Bu}_{-}} \boldsymbol{\kappa}^{2} N\right)\left(\mathrm{Me}_{2}-\mathrm{cod}\right)\right](14-\mathrm{sp})$

\begin{tabular}{|c|c|c|c|}
\hline H & -0.953476 & -4.775206 & \\
\hline & -2.339750 & -4.098435 & -0.082139 \\
\hline $\mathrm{H}$ & -0.714993 & -3.811055 & -0.714599 \\
\hline C & -2.424670 & -2.453827 & 1.968639 \\
\hline $\mathrm{H}$ & -3.314436 & -2.218448 & 1.381510 \\
\hline $\mathrm{H}$ & -2.620725 & -3.380124 & 2.519671 \\
\hline $\mathrm{H}$ & -2.273687 & -1.649007 & 2.694855 \\
\hline & -4.365452 & 0.296230 & -2.177496 \\
\hline $\mathrm{H}$ & -5.214173 & 0.373115 & -2.851145 \\
\hline$C$ & 2.472754 & -1.442280 & -0.774733 \\
\hline $\mathrm{C}$ & 3.300960 & -0.736581 & -1.844265 \\
\hline $\mathrm{H}$ & 4.289428 & -0.484042 & -1.451093 \\
\hline $\mathrm{H}$ & 3.481119 & -1.432732 & -2.671204 \\
\hline $\mathrm{C}$ & 2.592112 & 0.505348 & -2.390381 \\
\hline $\mathrm{H}$ & 1.861755 & 0.190757 & -3.145864 \\
\hline $\mathbf{H}$ & 3.304134 & 1.160345 & -2.918411 \\
\hline$C$ & 1.842356 & 1.320857 & -1.349033 \\
\hline $\mathrm{C}$ & 2.337116 & 1.588617 & -0.052814 \\
\hline $\mathrm{C}$ & 3.706533 & 1.174852 & 0.479114 \\
\hline $\mathrm{H}$ & 4.451886 & 1.203683 & -0.322740 \\
\hline $\mathrm{H}$ & 4.036833 & 1.920837 & 1.210250 \\
\hline C & 3.685020 & -0.210821 & 1.162298 \\
\hline $\mathrm{H}$ & 3.434981 & -0.074978 & 2.219930 \\
\hline $\mathrm{H}$ & 4.688356 & -0.666269 & 1.143350 \\
\hline $\mathrm{C}$ & 2.664517 & -1.175477 & 0.582070 \\
\hline $\mathrm{H}$ & 1.922280 & 2.466933 & 0.431475 \\
\hline $\mathrm{H}$ & 2.340030 & -1.934681 & 1.286904 \\
\hline $\mathrm{C}$ & -0.921495 & 2.361310 & 1.487963 \\
\hline $\mathrm{C}$ & -0.602810 & 3.775488 & 0.939870 \\
\hline $\mathrm{H}$ & 0.439472 & 3.865981 & 0.626177 \\
\hline $\mathrm{H}$ & -1.235001 & 4.054233 & 0.092529 \\
\hline $\mathrm{H}$ & -0.770470 & 4.516877 & 1.728934 \\
\hline $\mathrm{C}$ & -2.343965 & 2.374124 & 2.101291 \\
\hline $\mathrm{H}$ & -3.120250 & 2.657310 & 1.388999 \\
\hline $\mathrm{H}$ & -2.593240 & 1.385009 & 2.496975 \\
\hline $\mathrm{H}$ & -2.373477 & 3.090460 & 2.928723 \\
\hline $\mathrm{C}$ & 0.047181 & 2.068231 & 2.655753 \\
\hline $\mathrm{H}$ & -0.010284 & 2.866978 & 3.403810 \\
\hline $\mathrm{H}$ & -0.217789 & 1.122595 & 3.136685 \\
\hline $\mathrm{H}$ & 1.079004 & 1.988372 & 2.310685 \\
\hline $\mathrm{C}$ & -1.159388 & -2.621624 & 1.090083 \\
\hline $\mathrm{C}$ & 0.006079 & -2.865027 & 2.068279 \\
\hline $\mathrm{H}$ & 0.901086 & -3.223106 & 1.557781 \\
\hline $\mathrm{H}$ & 0.253711 & -1.946928 & 2.607499 \\
\hline $\mathrm{H}$ & -0.279361 & -3.633228 & 2.794474 \\
\hline $\mathrm{C}$ & 0.859457 & 2.267416 & -2.011997 \\
\hline $\mathrm{H}$ & 0.094082 & 1.721353 & -2.573096 \\
\hline $\mathrm{H}$ & 0.356626 & 2.919094 & -1.302690 \\
\hline $\mathrm{H}$ & 1.400685 & 2.898829 & -2.732380 \\
\hline $\mathrm{C}$ & 1.874021 & -2.749204 & -1.245908 \\
\hline $\mathrm{H}$ & 1.199098 & -2.599143 & -2.095864 \\
\hline $\mathrm{H}$ & 2.677771 & -3.418491 & -1.586840 \\
\hline $\mathrm{H}$ & 1.323232 & -3.263523 & -0.462050 \\
\hline
\end{tabular}




\section{[Rh(ATI $\left.\left.{ }^{t \mathrm{Bu} / \mathrm{Bu}^{\mathrm{Bu}}} \boldsymbol{\kappa}^{\mathbf{2}} N\right)\left(\mathrm{Me}_{2}-\mathrm{cod}\right)\right]$ (14-tet)}

$\mathrm{H}^{\circ}=-1194.521874$
$\mathrm{G}^{\circ}=-1194.611927$

\section{$\mathrm{Rh}$}

$\mathrm{N}$

C

C

C

$\mathrm{H}$

$\mathrm{H}$

$\mathrm{N}$

$\mathrm{N}$

C

$\mathrm{C}$

C

$\mathrm{H}$

$\mathrm{H}$

C

$\mathrm{H}$

$\mathrm{H}$

$\mathrm{H}$

C

$\mathrm{H}$

$\mathrm{H}$

$\mathrm{H}$

C

$\mathrm{H}$

C

$\mathrm{C}$

$\mathrm{H}$

$\mathrm{H}$

$\mathrm{C}$

$\mathrm{H}$

$\mathrm{H}$

C

C

$\mathrm{C}$

$\mathrm{H}$

$\mathrm{H}$

C

$\mathrm{H}$

$\mathrm{H}$

$\mathrm{C}$

$\mathrm{H}$

$\mathrm{H}$

C

C

$\mathrm{H}$

$\mathrm{H}$

$\mathrm{H}$

$\begin{array}{llll}\mathrm{C} & -2.006652 & -3.458848 & -0.933580 \\ \mathrm{H} & -2.958429 & -3.465125 & -0.403654\end{array}$

$\begin{array}{llll}\mathrm{H} & -2.139739 & -2.886659 & -1.857202\end{array}$

$\begin{array}{lrrr}\mathrm{H} & -1.773831 & -4.493078 & -1.208600 \\ \mathrm{C} & 0.433554 & -3.193423 & -0.941861 \\ \mathrm{H} & 0.593757 & -4.275113 & -1.008196 \\ \mathrm{H} & 0.314629 & -2.805954 & -1.958343 \\ \mathrm{H} & 1.313866 & -2.726994 & -0.507975 \\ \mathrm{C} & -1.021324 & 2.872719 & 0.320599 \\ \mathrm{C} & 0.180581 & 3.271811 & 1.200187 \\ \mathrm{H} & 0.154322 & 2.722918 & 2.146407 \\ \mathrm{H} & 1.126741 & 3.057016 & 0.710587 \\ \mathrm{H} & 0.143146 & 4.343979 & 1.420987 \\ \mathrm{C} & 1.199228 & 0.179267 & -2.849375 \\ \mathrm{H} & 0.785991 & -0.818384 & -3.022462 \\ \mathrm{H} & 0.370814 & 0.889408 & -2.787677 \\ \mathrm{H} & 1.803167 & 0.442242 & -3.731456 \\ \mathrm{C} & 2.521473 & -2.210862 & 1.788225 \\ \mathrm{H} & 2.295658 & -3.131670 & 1.245004 \\ \mathrm{H} & 3.477470 & -2.378213 & 2.309071 \\ \mathrm{H} & 1.758411 & -2.065713 & 2.558062\end{array}$

$\left[\operatorname{Rh}\left(\mathrm{ATI}^{t \mathrm{Bu} / t \mathrm{Bu}}-\mathrm{\kappa}^{5} \mathrm{C}\right)\left(\mathrm{Me}_{2}-\mathrm{cod}\right)\right](14-\mathrm{pi})$ 


$\begin{array}{lrcc}\mathrm{H} & -2.115716 & 2.418341 & 2.421610 \\ \mathrm{C} & -2.967967 & 2.193649 & 0.447868 \\ \mathrm{H} & -2.247323 & 2.971193 & 0.173052 \\ \mathrm{H} & -3.946622 & 2.696112 & 0.510243 \\ \mathrm{C} & -2.966822 & 1.169082 & -0.672376 \\ \mathrm{H} & -0.594552 & 0.667782 & 2.146167 \\ \mathrm{H} & -2.786251 & 1.593125 & -1.659804 \\ \mathrm{C} & 3.518236 & -2.415790 & 0.186213 \\ \mathrm{C} & 2.992509 & -3.463721 & -0.819374 \\ \mathrm{H} & 1.933801 & -3.691851 & -0.685060 \\ \mathrm{H} & 3.137877 & -3.112338 & -1.846227 \\ \mathrm{H} & 3.550675 & -4.399164 & -0.703273 \\ \mathrm{C} & 3.174414 & -2.813575 & 1.637359 \\ \mathrm{H} & 3.532725 & -2.048017 & 2.331946 \\ \mathrm{H} & 2.098412 & -2.935973 & 1.791604 \\ \mathrm{H} & 3.657694 & -3.762833 & 1.893319 \\ \mathrm{C} & 5.055383 & -2.352249 & 0.050545 \\ \mathrm{H} & 5.460847 & -1.599739 & 0.732183 \\ \mathrm{H} & 5.514508 & -3.321502 & 0.275523 \\ \mathrm{H} & 5.334562 & -2.063036 & -0.967497 \\ \mathrm{C} & -1.060196 & -1.922079 & 2.391318 \\ \mathrm{H} & -1.029313 & -2.886235 & 1.872154 \\ \mathrm{H} & -0.038962 & -1.558587 & 2.520843 \\ \mathrm{H} & -1.473489 & -2.113848 & 3.392481 \\ \mathrm{C} & -3.948022 & -0.789594 & -1.933399 \\ \mathrm{H} & -5.023300 & -0.642957 & -2.113545 \\ \mathrm{H} & -3.407909 & -0.391171 & -2.795433 \\ \mathrm{H} & -3.775256 & -1.870385 & -1.889552 \\ \mathrm{C} & 3.688549 & 3.399923 & 1.284641 \\ \mathrm{H} & 4.626352 & 2.921967 & 0.987757 \\ \mathrm{H} & 3.854289 & 4.479709 & 1.372843 \\ \mathrm{H} & 3.412995 & 3.005876 & 2.267489 \\ \mathrm{C} & 1.277198 & 3.749648 & 0.741697 \\ \mathrm{H} & 0.447552 & 3.639415 & 0.039628 \\ \mathrm{H} & 0.968748 & 3.311622 & 1.696635 \\ \mathrm{H} & 1.439995 & 4.821438 & 0.899470 \\ & & & \\ & & \end{array}$

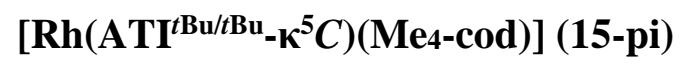

\begin{tabular}{|c|c|c|c|}
\hline & 2.609893 & 3.049209 & 0.491695 \\
\hline & 3.566237 & 3.573383 & -0.603067 \\
\hline & 3.824269 & 4.620327 & -0.409427 \\
\hline & 4.489444 & 2.986416 & -0.601140 \\
\hline & 3.135782 & 3.516832 & -1.606263 \\
\hline & -0.433844 & -0.372304 & -2.502489 \\
\hline & -1.136408 & -0.541593 & -3.312480 \\
\hline & -3.483337 & -0.073994 & -0.592360 \\
\hline$U$ & -4.204781 & -0.804469 & 0.543210 \\
\hline $\mathrm{F}$ & -4.660782 & -0.077180 & 1.219821 \\
\hline H & -5.043416 & -1.364480 & 0.114002 \\
\hline 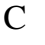 & -3.319407 & -1.799189 & 1.316629 \\
\hline $\mathrm{H}$ & -3.320027 & -2.751742 & 0.773631 \\
\hline 1 & -3.775595 & -2.019021 & 2.296157 \\
\hline 0 & -1.856269 & -1.409857 & 1.519292 \\
\hline$C$ & -1.456825 & -0.104738 & 1.891266 \\
\hline 0 & -2.503343 & 0.974169 & 2.200102 \\
\hline $\mathrm{H}$ & -3.396175 & 0.514837 & 2.632574 \\
\hline F & -2.104667 & 1.623587 & 2.987681 \\
\hline 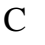 & -2.865502 & 1.862057 & 0.994428 \\
\hline 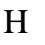 & -2.121803 & 2.664324 & 0.929361 \\
\hline H & -3.828475 & 2.368513 & 1.174450 \\
\hline 0 & -2.892618 & 1.182283 & -0.374876 \\
\hline C & 3.635530 & -2.386556 & -0.054761 \\
\hline C & 3.262474 & -3.293708 & -1.247783 \\
\hline $\mathrm{H}$ & 2.196462 & -3.523974 & -1.292273 \\
\hline 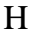 & 3.544682 & -2.812390 & -2.189902 \\
\hline t & 3.806903 & -4.241915 & -1.178882 \\
\hline$C$ & 3.165530 & -2.994106 & 1.283839 \\
\hline $\mathrm{H}$ & 3.393801 & -2.309831 & 2.106785 \\
\hline $\mathrm{H}$ & 2.092062 & -3.200439 & 1.298443 \\
\hline $\mathrm{H}$ & 3.685029 & -3.940048 & 1.472733 \\
\hline C & 5.174755 & -2.264402 & -0.011617 \\
\hline 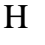 & 5.476966 & -1.612495 & 0.812731 \\
\hline $\mathrm{H}$ & 5.648708 & -3.243984 & 0.118050 \\
\hline $\mathrm{H}$ & 5.544256 & -1.818191 & -0.939973 \\
\hline & -1.033900 & -2.633903 & 1.885214 \\
\hline H & -1.084908 & -3.400495 & 1.104607 \\
\hline $\mathrm{H}$ & 0.012865 & -2.414312 & 2.086097 \\
\hline $\mathrm{H}$ & -1.454810 & -3.082745 & 2.797544 \\
\hline C & -3.931562 & -0.552041 & -1.961801 \\
\hline $\mathrm{H}$ & -4.991407 & -0.298472 & -2.116163 \\
\hline $\mathrm{H}$ & -3.363643 & -0.124153 & -2.786285 \\
\hline $\mathrm{H}$ & -3.851526 & -1.642110 & -2.030953 \\
\hline C & -0.114423 & 0.174150 & 2.542481 \\
\hline $\mathrm{H}$ & 0.226575 & 1.186530 & 2.307952 \\
\hline $\mathrm{H}$ & -0.204182 & 0.107829 & 3.637735 \\
\hline H & 0.678704 & -0.501745 & 2.225867 \\
\hline C & -2.730127 & 2.202061 & -1.490200 \\
\hline & -3.555752 & 2.927709 & -1.436823 \\
\hline & -1.799688 & 2.766160 & -1.378666 \\
\hline & -2.742295 & 1.767382 & -2.489342 \\
\hline & 1.274188 & 3.826738 & 0.493151 \\
\hline & 0.755564 & 3.791632 & -0.466550 \\
\hline
\end{tabular}




$\begin{array}{llll}\mathrm{H} & 0.601088 & 3.419738 & 1.255268 \\ \mathrm{H} & 1.456238 & 4.879130 & 0.737265 \\ \mathrm{C} & 3.286696 & 3.270591 & 1.862172 \\ \mathrm{H} & 4.232534 & 2.723791 & 1.905716 \\ \mathrm{H} & 3.482488 & 4.333525 & 2.043321 \\ \mathrm{H} & 2.647668 & 2.892144 & 2.666127\end{array}$

$\left[\operatorname{Rh}\left(\mathrm{ATI}^{t \mathrm{Bu} / t \mathrm{Bu}}-\boldsymbol{\kappa}^{2} N\right)\left(\mathrm{Me}_{4}-\operatorname{cod}\right)\right](15-$ tet $)$

$\mathrm{H}^{\circ}=-1 ' 273.077546$

$\mathrm{G}^{\circ}=-1273.172314$

$\begin{array}{llll}\mathrm{Rh} & -0.825720 & -0.028186 & 0.109392\end{array}$

$\begin{array}{lllll}\mathrm{N} & 0.985135 & 1.333323 & 0.617098\end{array}$

$\begin{array}{llll}\text { C } & 2.006218 & 0.764215 & -0.034131\end{array}$

$\begin{array}{llll}\text { C } & 2.813535 & 1.456798 & -0.987923\end{array}$

$\begin{array}{llll}\text { C } & 4.058500 & 1.188657 & -1.547841\end{array}$

$\begin{array}{llll}\mathrm{H} & 2.355380 & 2.372233 & -1.343794\end{array}$

$\begin{array}{llll}\mathrm{H} & 4.387319 & 1.909496 & -2.295546\end{array}$

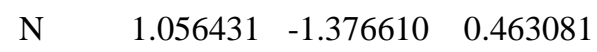

$\begin{array}{llll}\text { C } & 2.170312 & -0.709513 & 0.198251\end{array}$

$\begin{array}{llll}\text { C } & 4.699257 & -0.847630 & -0.317288\end{array}$

$\begin{array}{llll}\text { C } & 3.508680 & -1.224374 & 0.290516\end{array}$

$\begin{array}{llll}\mathrm{H} & 5.543406 & -1.4944440 & -0.080096\end{array}$

$\begin{array}{llll}\mathrm{H} & 3.608961 & -2.102587 & 0.909099\end{array}$

$\begin{array}{llll}\text { C } & 0.197812 & 3.647989 & 0.025399\end{array}$

$\begin{array}{llll}\mathrm{H} & 0.120921 & 4.675420 & 0.399559\end{array}$

$\begin{array}{lllll}\mathrm{H} & 0.661838 & 3.692377 & -0.965237\end{array}$

$\begin{array}{llll}\mathrm{H} & -0.811161 & 3.247103 & -0.093282\end{array}$

$\begin{array}{llll}\text { C } & 2.433867 & 3.384341 & 1.172653\end{array}$

$\begin{array}{llll}\mathrm{H} & 2.941149 & 3.623736 & 0.238448\end{array}$

$\begin{array}{llll}\mathrm{H} & 2.340787 & 4.315660 & 1.741346\end{array}$

$\begin{array}{llll}\mathrm{H} & 3.078590 & 2.707402 & 1.741067\end{array}$

$\begin{array}{llll}\text { C } & 4.960867 & 0.179465 & -1.226274\end{array}$

$\begin{array}{lllll}\mathrm{H} & 5.942331 & 0.205809 & -1.691324\end{array}$

$\begin{array}{llll}\text { C } & -2.764851 & 0.901499 & 0.462523\end{array}$

$\begin{array}{lllll}\text { C } & -3.447374 & 1.393987 & -0.824552\end{array}$

$\begin{array}{lllll}\mathrm{H} & -4.324159 & 0.777166 & -1.050283\end{array}$

$\begin{array}{llll}\mathrm{H} & -3.842543 & 2.400809 & -0.652686\end{array}$

$\begin{array}{llll}\text { C } & -2.469543 & 1.444855 & -2.001858\end{array}$

$\begin{array}{llll}\mathrm{H} & -2.014844 & 2.439802 & -2.035684\end{array}$

$\begin{array}{llll}\mathrm{H} & -2.987593 & 1.319740 & -2.967185\end{array}$

$\begin{array}{llll}\text { C } & -1.335559 & 0.430149 & -1.901951\end{array}$

$\begin{array}{llll}\text { C } & -1.600312 & -0.971950 & -1.672633\end{array}$

$\begin{array}{llll}\text { C } & -3.050847 & -1.467814 & -1.551863\end{array}$

$\begin{array}{llll}\mathrm{H} & -3.715032 & -0.840987 & -2.154790\end{array}$

$\begin{array}{llll}\mathrm{H} & -3.120791 & -2.469336 & -1.989487\end{array}$

$\begin{array}{llll}\text { C } & -3.500304 & -1.525252 & -0.086498\end{array}$

$\begin{array}{llll}\mathrm{H} & -3.274549 & -2.520489 & 0.308579\end{array}$

$\begin{array}{lllll}\mathrm{H} & -4.592842 & -1.406086 & 0.006414\end{array}$

$\begin{array}{llll}\text { C } & -2.785570 & -0.504699 & 0.796428\end{array}$

$\begin{array}{llll}\text { C } & 1.068872 & -2.799576 & 0.921535\end{array}$

$\begin{array}{llll}\text { C } & 1.756342 & -3.771463 & -0.069765\end{array}$

H $\quad 1.226559-3.773636 \quad-1.026156$

$\begin{array}{lllll}\mathrm{H} & 2.797923 & -3.525123 & -0.272080\end{array}$

$\begin{array}{lrrl}\mathrm{H} & 1.722919 & -4.790088 & 0.332144 \\ \mathrm{C} & 1.673005 & -2.908249 & 2.347357 \\ \mathrm{H} & 2.741148 & -2.697423 & 2.404477 \\ \mathrm{H} & 1.162459 & -2.206521 & 3.014255 \\ \mathrm{H} & 1.518769 & -3.920598 & 2.736128 \\ \mathrm{C} & -0.388302 & -3.269435 & 1.067395 \\ \mathrm{H} & -0.412770 & -4.321838 & 1.370518 \\ \mathrm{H} & -0.900385 & -2.684586 & 1.831428 \\ \mathrm{H} & -0.939088 & -3.168092 & 0.133394 \\ \mathrm{C} & 1.018114 & 2.776900 & 0.997299 \\ \mathrm{C} & 0.384454 & 2.825365 & 2.405694 \\ \mathrm{H} & -0.585027 & 2.335126 & 2.414025 \\ \mathrm{H} & 1.029761 & 2.305935 & 3.121240 \\ \mathrm{H} & 0.257138 & 3.860060 & 2.744465 \\ \mathrm{C} & -0.134382 & 0.839631 & -2.731222 \\ \mathrm{H} & 0.074884 & 1.905974 & -2.618096 \\ \mathrm{H} & 0.778719 & 0.297349 & -2.485429 \\ \mathrm{H} & -0.348517 & 0.657111 & -3.795695 \\ \mathrm{C} & -2.920649 & 1.922862 & 1.578323 \\ \mathrm{H} & -2.538490 & 2.904238 & 1.286199 \\ \mathrm{H} & -3.994410 & 2.051126 & 1.790143 \\ \mathrm{H} & -2.445560 & 1.638754 & 2.516553 \\ \mathrm{C} & -2.791593 & -0.888159 & 2.270762 \\ \mathrm{H} & -2.752470 & -1.971457 & 2.401360 \\ \mathrm{H} & -1.953244 & -0.454769 & 2.826233 \\ \mathrm{H} & -3.712744 & -0.532662 & 2.755862 \\ \mathrm{C} & -0.686779 & -1.997815 & -2.323449 \\ \mathrm{H} & 0.372169 & -1.770959 & -2.211288 \\ \mathrm{H} & -0.860785 & -2.997602 & -1.919669 \\ \mathrm{H} & -0.912292 & -2.046455 & -3.400360\end{array}$

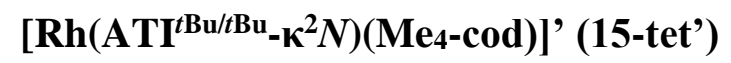




$\begin{array}{lrrr}\mathrm{H} & -0.664407 & -4.670950 & 0.772326 \\ \mathrm{H} & -0.819516 & -3.213754 & 1.753784 \\ \mathrm{C} & -5.335247 & -0.040347 & 0.155001 \\ \mathrm{H} & -6.419651 & -0.047113 & 0.222255 \\ \mathrm{C} & 2.435962 & 1.087531 & -0.987508 \\ \mathrm{C} & 3.573581 & 1.465950 & -0.023353 \\ \mathrm{H} & 4.475128 & 0.888841 & -0.258025 \\ \mathrm{H} & 3.849499 & 2.511960 & -0.194685 \\ \mathrm{C} & 3.162655 & 1.294272 & 1.445910 \\ \mathrm{H} & 2.786637 & 2.250270 & 1.820864 \\ \mathrm{H} & 4.029998 & 1.046155 & 2.079968 \\ \mathrm{C} & 2.066701 & 0.250950 & 1.649631 \\ \mathrm{C} & 2.235552 & -1.104897 & 1.179894 \\ \mathrm{C} & 3.517500 & -1.494396 & 0.422739 \\ \mathrm{H} & 4.369450 & -0.921881 & 0.805512 \\ \mathrm{H} & 3.753692 & -2.541514 & 0.640924 \\ \mathrm{C} & 3.360423 & -1.325874 & -1.094017 \\ \mathrm{H} & 3.033367 & -2.278562 & -1.520503 \\ \mathrm{H} & 4.325857 & -1.098453 & -1.575997 \\ \mathrm{C} & 2.331795 & -0.265845 & -1.479166 \\ \mathrm{C} & -1.106689 & 2.918476 & 0.150482 \\ \mathrm{C} & -1.132547 & 3.636229 & -1.221444 \\ \mathrm{H} & -0.380225 & 3.223536 & -1.893383 \\ \mathrm{H} & -2.100198 & 3.572644 & -1.723635 \\ \mathrm{H} & -0.916898 & 4.701961 & -1.086001 \\ \mathrm{C} & -2.277311 & 3.421983 & 1.032012 \\ \mathrm{H} & -3.257402 & 3.309673 & 0.569903\end{array}$

$\begin{array}{crrr}\mathrm{H} & -2.291139 & 2.882573 & 1.984503 \\ \mathrm{H} & -2.129508 & 4.485517 & 1.248540 \\ \mathrm{C} & 0.173310 & 3.344233 & 0.891074 \\ \mathrm{H} & 0.248261 & 4.436355 & 0.925940 \\ \mathrm{H} & 0.151483 & 2.975103 & 1.918799 \\ \mathrm{H} & 1.061041 & 2.943890 & 0.408100 \\ \mathrm{C} & -1.069840 & -2.868762 & -0.412789 \\ \mathrm{C} & 0.242814 & -3.257154 & -1.111884 \\ \mathrm{H} & 0.320711 & -2.744278 & -2.071893 \\ \mathrm{H} & 1.111032 & -2.990258 & -0.511857 \\ \mathrm{H} & 0.263119 & -4.335893 & -1.300490 \\ \mathrm{C} & 1.260536 & 0.485160 & 2.919432 \\ \mathrm{H} & 1.079954 & 1.548781 & 3.082098 \\ \mathrm{H} & 0.291098 & -0.018258 & 2.908461 \\ \mathrm{H} & 1.815293 & 0.108156 & 3.792268 \\ \mathrm{C} & 2.101109 & 2.219727 & -1.946187 \\ \mathrm{H} & 1.959606 & 3.169331 & -1.426405 \\ \mathrm{H} & 2.944783 & 2.363189 & -2.640752 \\ \mathrm{H} & 1.215511 & 2.029631 & -2.552203 \\ \mathrm{C} & 1.728276 & -2.240410 & 2.052539 \\ \mathrm{H} & 0.768488 & -2.033903 & 2.523528 \\ \mathrm{H} & 1.639084 & -3.174918 & 1.495222 \\ \mathrm{H} & 2.456534 & -2.420283 & 2.859719 \\ \mathrm{C} & 1.748030 & -0.483073 & -2.868989 \\ \mathrm{H} & 2.399448 & -0.030814 & -3.631639 \\ \mathrm{H} & 1.663098 & -1.545695 & -3.101650 \\ \mathrm{H} & 0.756490 & -0.036563 & -2.985610\end{array}$




\section{References}

1 Gaussian 09, Revision or B.01 or D.01, Frisch, M. J.; Trucks, G. W.; Schlegel, H. B.; Scuseria, G. E.; Robb, M. A.; Cheeseman, J. R.; Scalmani, G.; Barone, V.; Mennucci, B.; Petersson, G. A.; Nakatsuji, H.; Caricato, M.; Li, X.; Hratchian, H. P.; Izmaylov, A. F.; Bloino, J.; Zheng, G.; Sonnenberg, J. L.; Hada, M.; Ehara, M.; Toyota, K.; Fukuda, R.; Hasegawa, J.; Ishida, M.; Nakajima, T.; Honda, Y.; Kitao, O.; Nakai, H.; Vreven, T.; Montgomery, Jr., J. A.; Peralta, J. E.; Ogliaro, F.; Bearpark, M.; Heyd, J. J.; Brothers, E.; Kudin, K. N.; Staroverov, V. N.; Kobayashi, R.; Normand, J.; Raghavachari, K.; Rendell, A.; Burant, J. C.; Iyengar, S. S.; Tomasi, J.; Cossi, M.; Rega, N.; Millam, J. M.; Klene, M.; Knox, J. E.; Cross, J. B.; Bakken, V.; Adamo, C.; Jaramillo, J.; Gomperts, R.; Stratmann, R. E.; Yazyev, O.; Austin, A. J.; Cammi, R.; Pomelli, C.; Ochterski, J. W.; Martin, R. L.; Morokuma, K.; Zakrzewski, V. G.; Voth, G. A.; Salvador, P.; Dannenberg, J. J.; Dapprich, S.; Daniels, A. D.; Farkas, Ö.; Foresman, J. B.; Ortiz, J. V.; Cioslowski, J.; Fox, D. J. Gaussian, Inc., Wallingford CT, 2009.

2 Hariharan, P. C.; Pople, J. A. Theor. Chim. Acta 1973, 28, 213-222.

3 (a) Dunning Jr., T. H.; Hay, P. J. in Modern Theoretical Chemistry, Ed. Schaefer III, H. F.; Vol. 3 (Plenum, New York, 1977) 1-28 (b) Hay, P. J.; Wadt, W. R. J. Chem. Phys. 1985, 82, 270-283; (c) Hay, P. J.; Wadt, W. R. J. Chem. Phys., 82 (1985) 299310.

$4 \quad$ Becke, A. D. J. Chem. Phys., 1993, 98, 5648-5652.

5 These calculations were performed at a lower level of theory compared to the rest of the calculations in this work and should give qualitative indications for the relative energies of the calculated compounds.

6 For comparison: 5 is favored over 5-pi by $\Delta \mathrm{H}_{\mathrm{rel}}=-28.2 \mathrm{kcal} \cdot \mathrm{mol}^{-1}$ and $\Delta \mathrm{G}_{\mathrm{rel}}=-26.0$ $\mathrm{kcal} \cdot \mathrm{mol}^{-1}$ according to DFT calculations using the B3LYP functional and the 6$31 \mathrm{G}(\mathrm{d}, \mathrm{p})(\mathrm{C}, \mathrm{H}, \mathrm{N})$ and LanL2DZ $(\mathrm{Rh})$ basis sets. 